\title{
Pulse-driven quantum dynamics beyond the impulsive regime
}

\author{
D. Daems* \\ Center for Nonlinear Phenomena and Complex Systems, \\ Université Libre de Bruxelles, CP 231, 1050 Brussels, Belgium \\ S. Guérin $\dagger$ and H. R. Jauslin \\ Laboratoire de Physique de l'Université de Bourgogne, \\ UMR CNRS 5027, BP 47870, 21078 Dijon, France \\ A. Kellert and O. Atabek \\ Laboratoire de Photophysique Moléculaire du CNRS, Université Paris-Sud, \\ Bât. 210 - Campus d'Orsay, 91405 Orsay Cedex, France
}

\begin{abstract}
We review various unitary time-dependent perturbation theories and compare them formally and numerically. We show that the Kolmogorov-Arnold-Moser technique performs better owing to both the superexponential character of correction terms and the possibility to optimize the accuracy of a given level of approximation which is explored in details here. As an illustration, we consider a two-level system driven by short pulses beyond the sudden limit.

PACS numbers: $42.50 . \mathrm{Hz}, 02.30 . \mathrm{Mv}, 03.65 .-\mathrm{w}$
\end{abstract}

\section{INTRODUCTION}

Short and intense laser pulses allow nowadays to drive atoms and molecules in nonperturbative regimes going from adiabatic (nano- and picosecond) to sudden or impulsive (femtosecond). Recent examples concern the alignment of molecules, which can be achieved during nanosecond pulses or after femtosecond pulses [1]. Corrections to perfect adiabaticity can be analyzed in terms of superadiabatic 2, 3, 4, 5] and Davis-Dykhne-Pechukas techniques [6, 7, 8]. On the opposite side, regimes beyond the impulsive approximation, i.e. beyond the limiting case of pulses described as $\delta$-kicks, have not been yet much explored due to a lack of adapted tools of analysis.

It is well-known that one can treat periodic perturbations using extended Hilbert spaces where time is considered as a new dynamical variable, in order to render the problem autonomous 9, 10]. This approach, that can be formulated as Floquet theory [11, 12, 13], allows one to eliminate systematically secular terms (i.e. terms that grow arbitrary with time), which would otherwise lead to divergences. Pulse-driven dynamics associated to Hamiltonians localized in time requires a different treatment of secular terms. In this case, since the perturbation acts only during a finite time interval, the secular terms do not lead to divergences.

This article contributes to develop a time-dependent perturbation technique, that is in particular suited for pulse-driven dynamics, on the basis of Refs. 14, 15]. In Ref. 14], we constructed a superexponential perturbation theory which preserves the unitarity of the evolu-

\footnotetext{
*Electronic address: ddaems@ulb.ac.be

${ }^{\dagger}$ Electronic address: sguerin@u-bourgogne.fr

${ }^{\ddagger}$ Electronic address: arne.keller@ppm.u-psud.fr
}

tion operator at each order, and applied it beyond the impulsive regime by considering an expansion where the perturbative parameter is the characteristic duration of the time-dependent interaction compared to the characteristic time for the free evolution. We have shown that it converges in any regime (from impulsive to adiabatic) in two-level systems. This derivation is based on the Kolmogorov-Arnold-Moser (KAM) technique applied in an extended Hilbert space [16, 17, 18, 19, 20]. In Ref. [15], we presented an improvment of this technique taking advantage of free parameters, connected to secular terms, that are available to reduce the error without prior knowledge of the exact solution. This optimization enhances the accuracy of the method in such a way that the first order approximation gives a satisfactory description up to fairly large values of the perturbative parameter.

The present article contains a detailed description of the methods announced in [15]. Instead of using an extended space, we formulate the derivation in a simpler way, by stating the perturbation iterations directly at the level of the evolution operator in the original Hilbert space. This scheme allows us to consider and compare in a unified way various time-dependent perturbation techniques. In particular we make the connection with the well-known Magnus expansion 21], that has been used by Henriksen et al. to construct an improved impulsive approximation [22]. We also develop and investigate the accuracy optimization which can be applied to the timedependent Poincaré-Von Zeipel, the time-dependent Van Vleck and the time-dependent KAM techniques.

The paper is organized as follows. In Sec. II we recall the Magnus expansion and outline the time-dependent versions of the Poincaré-Von Zeipel, the Van Vleck and the KAM techniques. We highlight the free parameters and free operators that may be present in these unitary perturbative methods. In Sec. III we exploit these degrees of freedom to improve the accuracy of a given level 
of approximation. Section $\square$ is devoted to the application of these techniques beyond the impulsive regime and the illustration on a pulse-driven two-level system. The conclusions are given in Sec. $\mathbb{\nabla}$ and some details of the calculations are reported in Appendixes A to C.

\section{UNITARY TIME-DEPENDENT PERTURBATION THEORIES}

We consider the Schrödinger equation

$i \frac{\partial}{\partial t} U_{H_{1}}\left(t, t_{0}\right)=H_{1}(t) U_{H_{1}}\left(t, t_{0}\right), \quad U_{H_{1}}\left(t_{0}, t_{0}\right)=\mathbb{1}$,

where $H_{1}(t)$ is a time-dependent matrix or a timedependent operator in a Hilbert space $\mathbb{H}$. Assuming that one can decompose $H_{1}(t)$ according to

$$
H_{1}(t)=H_{0}(t)+\epsilon V_{1}(t),
$$

where $H_{0}(t)$ is such that its propagator $U_{H_{0}}\left(t, t_{0}\right)$ is known and $V_{1}(t)$ is localized in time (i.e., vanishes outside a finite interval), we are looking for a unitary perturbative expansion of the full propagator $U_{H_{1}}\left(t, t_{0}\right)$. This can be achieved by two classes of techniques that we outline below: i) the order by order methods, namely the Magnus expansion, the time-dependent Poincaré-Von Zeipel technique and the time-dependent Van Vleck technique, where after $n$ steps the remainder is of order $\epsilon^{n+1}$; and ii) the surperexponential KAM technique where the remainder is of order $\epsilon^{2^{n-1}}$.

Below we will have to consider the propagator $U_{H_{1}}\left(t, t_{0}\right)$ in the interaction representation with respect to $H_{0}(t)$

$$
U_{H_{1}^{\mathrm{i}}}\left(t, t_{0} ; s\right) \equiv U_{H_{0}}(s, t) U_{H_{1}}\left(t, t_{0}\right) U_{H_{0}}\left(t_{0}, s\right),
$$

where $s$ is an arbitrary time (the standard interaction representation corresponds to the case $s=t_{0}$ ). This propagator satisfies the Schrödinger equation

$$
i \frac{\partial}{\partial t} U_{H_{1}^{\mathrm{i}}}\left(t, t_{0} ; s\right)=H_{1}^{\mathrm{i}}(t ; s) U_{H_{1}^{\mathrm{i}}}\left(t, t_{0} ; s\right),
$$

and the associated Hamiltonian reads

$$
H_{1}^{\mathrm{i}}(t ; s) \equiv \epsilon U_{H_{0}}(s, t) V_{1}(t) U_{H_{0}}(t, s) .
$$

We will also consider a new representation defined with the help of a unitary transformation $T(t ; s)$ according to

$$
U_{H}\left(t, t_{0} ; s\right) \equiv T^{\dagger}(t ; s) U_{H_{1}}\left(t, t_{0}\right) T\left(t_{0}, s\right),
$$

where $H(t ; s)$ is a new Hamiltonian. This expression is reminiscent of Eq. (2a) although $T(t ; s)$ need not be a propagator but a unitary operator which features an arbitrary parameter $s$ and satisfies the property $T^{\dagger}(t ; s)=$ $T(s ; t)$.

\section{A. Magnus expansion}

The solution to Eq. (1a) can always be put in the form of an exponential

$$
U_{H_{1}}\left(t, t_{0}\right)=e^{-i M_{H_{1}}\left(t ; t_{0}\right)}, \quad M_{H_{1}}\left(t_{0} ; t_{0}\right)=0,
$$

where $M_{H_{1}}\left(t ; t_{0}\right)$ is self-adjoint to ensure unitarity. The exponent $M_{H_{1}}\left(t ; t_{0}\right)$ is generally not simply the integral of $H_{1}(t)$ owing to the non-commutativity of this latter for different times. Indeed from Eq. (1a) one deduces the following equation for $M_{H_{1}}\left(t ; t_{0}\right)$

$$
\frac{\partial}{\partial t} M_{H_{1}}\left(t ; t_{0}\right)=H_{1}(t)+\frac{i}{2}\left[M_{H_{1}}\left(t ; t_{0}\right), H_{1}(t)\right]-\frac{1}{12}\left[M_{H_{1}}\left(t ; t_{0}\right),\left[M_{H_{1}}\left(t ; t_{0}\right), H_{1}(t)\right]\right]+\cdots .
$$

We refer to the paper of Magnus 21] for a derivation of this equation (see also Ref. 23]). The matrix or the operator $M_{H_{1}}\left(t ; t_{0}\right)$ is obtained by integrating the first term on the right hand side of Eq. (55) and substituting the result into the next terms of this equation. One then repeats this procedure, known as Picard's iteration, with the resulting second and subsequent terms. This yields the Magnus expansion

$$
\begin{aligned}
M_{H_{1}}\left(t ; t_{0}\right) & =\int_{t_{0}}^{t} d u H_{1}(u)+\frac{i}{2} \int_{t_{0}}^{t} d u\left[\int_{t_{0}}^{u} d v H_{1}(v), H_{1}(u)\right]-\frac{1}{4} \int_{t_{0}}^{t} d u\left[\int_{t_{0}}^{u} d v\left[\int_{t_{0}}^{v} d w H_{1}(w), H_{1}(v)\right], H_{1}(u)\right] \\
& -\frac{1}{12} \int_{t_{0}}^{t} d u\left[\int_{t_{0}}^{u} d v H_{1}(v),\left[\int_{t_{0}}^{u} d w H_{1}(w), H_{1}(u)\right]\right]+\cdots
\end{aligned}
$$

The number of terms in this expansion grows very rapidly 24]. Notice that this expansion is not limited to perturbation theory [i.e. to a Hamiltonian of the form of
Eq. [1b] although it is of interest only when the subsequent terms are negligible. This will be the case if one is interested in obtaining an expression valid for very short 
times only or in the presence of another small parameter.

In the framework of perturbation theory, if we were to substitute Eq. (1b) into Eq. (6) we would generally obtain contributions to a given order in $\epsilon$ from an infinite series of terms of the Magnus expansion [33]. This can easily be circumvented by considering Eq. (2), the equivalent of Eq. (1) in the interaction representation. The Magnus expansion pertaining to Eq. (2b) allows one to write its solution in the form

$$
U_{H_{1}^{\mathrm{i}}}\left(t, t_{0} ; s\right)=\exp \left\{-i \epsilon M_{H_{1}^{\mathrm{i}}}\left(t ; t_{0}, s\right)\right\},
$$

with $\epsilon M_{H_{1}^{\mathrm{i}}}\left(t ; t_{0}, s\right)$ given by Eq. (6) where $H_{1}(t)$ is replaced by $H_{1}^{\mathrm{i}}(u ; s)$. By virtue of Eq. (2C), each $H_{1}^{\mathrm{i}}(u ; s)$ carries a prefactor $\epsilon$. Hence, there is no $\epsilon$-independent term and terms of $M_{H_{1}^{\mathrm{i}}}\left(t ; t_{0}, s\right)$ with higher numbers of $H_{1}^{\mathrm{i}}(u ; s)$ are now of higher orders in $\epsilon$. In order to display the explicit dependence on the parameter $s$, we decompose the propagator entering through $H_{1}^{\mathrm{i}}(u ; s)$ according to $U_{H_{0}}(s, u)=U_{H_{0}}(s, t) U_{H_{0}}(t, u)$ and note that the leftmost $U_{H_{0}}(s, t)$ and rightmost $U_{H_{0}}(t, s)$ of each term of $M_{H_{1}^{\mathrm{i}}}\left(t ; t_{0}, s\right)$ factor out (while the inner ones cancel each other)

$$
\epsilon M_{H_{1}^{\mathrm{i}}}\left(t ; t_{0}, s\right)=U_{H_{0}}(s, t) \sum_{k=1}^{\infty} \epsilon^{k} M_{k}\left(t ; t_{0}\right) U_{H_{0}}(t, s) .
$$

Returning to the original representation with the help of Eq. (2a), Eq. (7) yields

$$
U_{H_{1}}\left(t, t_{0}\right)=\exp \left\{-i \sum_{k=1}^{\infty} \epsilon^{k} M_{k}\left(t ; t_{0}\right)\right\} U_{H_{0}}\left(t, t_{0}\right),
$$

where use was made of the identity

$$
A e^{B} A^{-1}=e^{A B A^{-1}} .
$$

By truncating the infinite series of Eq. (9) to order $n$ one obtains the unitary approximation

$$
U_{H_{1}}\left(t, t_{0}\right)=U_{H_{1}}^{(n)}\left(t, t_{0}\right)+\mathcal{O}\left(\epsilon^{n+1}\right),
$$

with the Magnus expansion

$$
U_{H_{1}}^{(n)}\left(t, t_{0}\right)=\exp \left\{-i \sum_{k=1}^{n} \epsilon^{k} M_{k}\left(t ; t_{0}\right)\right\} U_{H_{0}}\left(t, t_{0}\right) .
$$

We stress that this expression is independent of the arbitrary time $s$ chosen in Eq. (2) 34]. Each term of the exponential can be cast into the form

$$
M_{k}\left(t ; t_{0}\right)=\int_{t_{0}}^{t} d u U_{H_{0}}(t, u) V_{k}(u) U_{H_{0}}(u, t),
$$

where $V_{k}(u)$ is deduced from Eqs. (2c), (6) and (8). The case $k=1$ features the perturbation itself while for the first few values of $k$ that we shall use below, dropping the time arguments, one finds

$$
\begin{aligned}
V_{2} & =-\frac{i}{2}\left[M_{1}, V_{1}\right], \\
V_{3} & =-i\left[M_{2}, V_{1}\right]+\frac{1}{6}\left[M_{1},\left[M_{1}, V_{1}\right]\right] .
\end{aligned}
$$

\section{B. Time-dependent Poincaré-Von Zeipel expansion}

The classical Poincaré-Von Zeipel technique was adapted to quantum mechanics by Scherer to treat both time-independent 25] and time-dependent 20] sytems. In the time-independent case it was shown 25] that this technique coincides with the usual Rayleigh-Schrödinger expansion. We present in Appendix $\mathrm{A}$ a time-dependent Poincaré-Von Zeipel technique that has the advantages to be strictly unitary upon truncation at any given order and does not require the consideration of a so-called extended Hilbert space. This method amounts to map the full propagator $U_{H_{1}}\left(t, t_{0}\right)$ into a new effective propagator $U_{H^{\mathrm{e}}}\left(t, t_{0}\right)$ with the help of a single unitary transformation according to an equation similar to Eq. (3). Moreover, this formulation exhibits free parameters available to improve the accuracy of a given step of the algorithm by a procedure we describe in Sec. IIIB Finally, we show that the time-dependent Poincaré-Von Zeipel method includes the Magnus expansion as a particular case.

\section{Time-dependent Van Vleck technique}

To provide a general perspective of unitary timedependent perturbation theories we introduce in Appendix $B$ a time-dependent version of the Van Vleck technique that is more widely used than the preceding method in the stationary case [31]. It consists in transforming the full propagator into a new effective propagator iteratively through a series of unitary operations. Our purpose is actually not to introduce yet another variant of perturbation theory but to emphasize that this timedependent version of a well-known technique is i) comparable to the Poincaré-Von Zeipel method which we show to be closely related to the Magnus expansion, and ii) not as performant as the KAM technique detailed below since it is an order by order perturbative method.

\section{Time-dependent Kolmogorov-Arnold-Moser expansion}

The time-dependent KAM technique aims at obtaining a superexponential expansion for the propagator $U_{H_{1}}\left(t, t_{0}\right)$ through a series of unitary transformations. It is sometimes said to be superconvergent. However, superexponential is more appropriate since the dependence on $\epsilon$ of the remainder after $n$ iterations is indeed the exponential of an exponential $\left(\epsilon^{2^{n-1}}\right)$ while the actual convergence of the algorithm has to be examined specifically.

\section{First iteration}

The first step is to construct a unitary operator $T_{1}(t)$ which transforms the propagator $U_{H_{1}}\left(t, t_{0}\right)$ we are look- 
ing for into the propagator $U_{H_{2}}\left(t, t_{0}\right)$ [cf. Eq. (3)]

$$
T_{1}^{\dagger}(t) U_{H_{1}}\left(t, t_{0}\right) T_{1}\left(t_{0}\right)=U_{H_{2}}\left(t, t_{0}\right),
$$

where $U_{H_{2}}\left(t, t_{0}\right)$ is associated with the sum of an effective Hamiltonian $H_{1}^{\mathrm{e}}(t)$ which contains all contributions up to order $\epsilon$ and a remainder $\epsilon^{2} V_{2}(t)$

$$
H_{2}(t) \equiv H_{1}^{\mathrm{e}}(t)+\epsilon^{2} V_{2}(t) .
$$

As the new propagator is generated by a sum of Hamiltonians it can be expressed in terms of the propagator $U_{H_{1}^{\mathrm{e}}}\left(t, t_{0}\right)$ and a propagator $U_{R_{2}}\left(t, t_{0}\right)$ by considering the interaction representation with respect to $H_{1}^{\mathrm{e}}\left(t ; t_{1}\right)$

$$
U_{H_{2}}\left(t, t_{0}\right)=U_{H_{1}^{\mathrm{e}}}\left(t, t_{1}^{\prime \prime}\right) U_{R_{2}}\left(t, t_{0} ; t_{1}^{\prime \prime}\right) U_{H_{1}^{\mathrm{e}}}\left(t_{1}^{\prime \prime}, t_{0}\right) .
$$

By virtue of Eq. (2) the propagator $U_{R_{2}}\left(t, t_{0} ; t_{1}^{\prime \prime}\right)$ satisfies a Schrödinger equation whose Hamiltonian is

$$
\epsilon^{2} R_{2}\left(t ; t_{1}^{\prime \prime}\right) \equiv \epsilon^{2} U_{H_{1}^{\mathrm{e}}}\left(t_{1}^{\prime \prime}, t\right) V_{2}(t) U_{H_{1}^{\mathrm{e}}}\left(t, t_{1}^{\prime \prime}\right)
$$

Being associated to a Hamiltonian of second order in $\epsilon$, this propagator will be neglected in Eq. (17), i.e., replaced by the identity.

The effective Hamiltonian can be decomposed as

$$
H_{1}^{\mathrm{e}}(t) \equiv H_{0}(t)+\epsilon D_{1}(t)
$$

which allows one to express $U_{H_{1}^{\mathrm{e}}}\left(t, t_{0}\right)$ as

$$
U_{H_{1}^{\mathrm{e}}}\left(t, t_{0}\right)=U_{H_{0}}\left(t, t_{1}\right) U_{P_{1}}\left(t, t_{0} ; t_{1}\right) U_{H_{0}}\left(t_{1}, t_{0}\right),
$$

where $U_{P_{1}}\left(t, t_{0}\right)$ is the propagator corresponding to the Hamiltonian

$$
\epsilon P_{1}\left(t ; t_{1}\right) \equiv \epsilon U_{H_{0}}\left(t_{1}, t\right) D_{1}(t) U_{H_{0}}\left(t, t_{1}\right) .
$$

Thus far the only restriction on the Hamiltonian $\epsilon D_{1}(t)$ is that it be of order $\epsilon$. Hence we have the freedom to choose it so as to be able to determine explicitly the propagator $U_{P_{1}}\left(t, t_{0} ; t_{1}\right)$. This will be the case if

$$
D_{1}(t) \equiv U_{H_{0}}\left(t, t_{1}\right) D_{1}\left(t_{1} ; t_{1}\right) U_{H_{0}}\left(t_{1}, t\right) \equiv D_{1}\left(t ; t_{1}\right),
$$

with $D_{1}\left(t_{1} ; t_{1}\right)$ arbitrary. In this paper, we consider explicitly two possibilities

$$
\begin{aligned}
& D_{1}\left(t_{1} ; t_{1}\right) \equiv 0, \\
& D_{1}\left(t_{1} ; t_{1}\right) \equiv V_{1}\left(t_{1}\right) .
\end{aligned}
$$

The first one is a trivial choice which gives nevertheless a nontrivial one-iteration KAM expansion (it will be shown in Sec. ID 4 to coincide, for the first iteration only, with the first order Magnus expansion). The choice to relate $D_{1}\left(t_{1} ; t_{1}\right)$ to the perturbation according to Eq. (23b) is also rather natural as this operator enters the effective Hamiltonian (we shall discuss the fact that the perturbation is evaluated at an arbitrary time $t_{1}$ in Sec. IIIA). Choosing one of the possibilities of Eq. (23) implies that the propagator defined in Eq. (20) reads

$$
U_{P_{1}}\left(t, t_{0} ; t_{1}\right)=e^{-i\left(t-t_{0}\right) \epsilon D_{1}\left(t_{1} ; t_{1}\right)} .
$$

The effective propagator can then be given a convenient form using Eqs. (10), (20) and (22)

$$
\begin{aligned}
U_{H_{1}^{\mathrm{e}}}\left(t, t_{0}\right) & =U_{H_{0}}\left(t, t_{0}\right) e^{-i\left(t-t_{0}\right) \epsilon D_{1}\left(t_{0} ; t_{1}\right)} \\
& \equiv U_{H_{1}^{\mathrm{e}}}\left(t, t_{0} ; t_{1}\right) .
\end{aligned}
$$

We now express the requirement that $T_{1}(t)$ defined in Eq. (15) be such that $\epsilon^{2} V_{2}(t)$ contain no terms of order lower than $\epsilon^{2}$. We first multiply Eq. (1a) from the left by $T_{1}^{\dagger}(t)$ and from the right by $T_{1}\left(t_{0}\right)$ to deduce employing also Eq. (15)

$\epsilon^{2} V_{2}(t)=T_{1}^{\dagger}(t) H_{1}(t) T_{1}(t)-H_{1}^{\mathrm{e}}(t)-T_{1}^{\dagger}(t) i \frac{\partial}{\partial t} T_{1}(t)$

Writing $T_{1}(t)$ in the exponential form

$$
T_{1}(t) \equiv e^{-i \epsilon W_{1}(t)},
$$

we then require that all terms of order lower than $\epsilon^{2}$ in Eq. (26) vanish identically. This leads to a differential equation for the self-adjoint operator $W_{1}(t)$

$$
\frac{\partial}{\partial t} W_{1}(t)=V_{1}(t)-D_{1}\left(t ; t_{1}\right)+i\left[W_{1}(t), H_{0}(t)\right],
$$

and defines the remainder $\epsilon^{2} V_{2}(t)$ as the right side of Eq. (26). We stress that to arrive at Eq. (28) we do not identify terms order by order. Hence this equation is still valid if any of the operator featured contains a further dependence on $\epsilon$. The general solution to Eq. (28) reads

$$
\begin{aligned}
W_{1}(t) & =\int_{t_{1}^{\prime}}^{t} d u U_{H_{0}}(t, u)\left[V_{1}(u)-D_{1}\left(u ; t_{1}\right)\right] U_{H_{0}}(u, t) \\
& +U_{H_{0}}\left(t, t_{0}\right) B_{1} U_{H_{0}}\left(t_{0}, t\right)
\end{aligned}
$$

where $B_{1}$ is any constant self-adjoint operator. In the present work we choose $B_{1}=0$.

Substituting Eq. (17) into Eq. (15) and replacing $U_{R_{2}}\left(t, t_{0} ; t_{1}^{\prime \prime}\right)$ by the identity as discussed above, one obtains the KAM approximation

$$
U_{H_{1}}\left(t, t_{0}\right)=U_{H_{1}}^{(1)}\left(t, t_{0}\right)+\mathcal{O}\left(\epsilon^{2}\right),
$$

with the one-iteration KAM expansion

$$
U_{H_{1}}^{(1)}\left(t, t_{0}\right)=T_{1}(t) U_{H_{1}^{\mathrm{e}}}\left(t, t_{0}\right) T_{1}^{\dagger}\left(t_{0}\right) .
$$

We emphasize the dependence of the following operators on the arbitrary times $t_{1}, t_{1}^{\prime}$ and $t_{1}^{\prime \prime}$

$$
\begin{aligned}
H_{1}^{\mathrm{e}}(t) & \equiv H_{1}^{\mathrm{e}}\left(t ; t_{1}\right), \\
T_{1}(t) & \equiv T_{1}\left(t ; t_{1}, t_{1}^{\prime}\right), \\
W_{1}(t) & \equiv W_{1}\left(t ; t_{1}, t_{1}^{\prime}\right), \\
V_{2}(t) & \equiv V_{2}\left(t ; t_{1}, t_{1}^{\prime}\right), \\
R_{2}\left(t ; t_{1}^{\prime \prime}\right) & \equiv R_{2}\left(t ; t_{1}, t_{1}^{\prime}, t_{1}^{\prime \prime}\right) .
\end{aligned}
$$

As a consequence, the one-iteration KAM expansion depends on $t_{1}$ and $t_{1}^{\prime}$. In Sec.IIIB we shall show how these parameters can be chosen to improve the accuracy of the algorithm. In addition, recall that there are two constant operators, $D_{1}\left(t_{1} ; t_{1}\right)$ in Eq. (22) and $B_{1}$ in Eq. (29), that can be freely chosen. Note that with the choice of Eq. 23a there is no dependence on $t_{1}$. 


\section{Second iteration}

In the first iteration of the KAM algorithm we started with the Hamiltonian $H_{1}(t)=H_{0}(t)+\epsilon V_{1}(t)$ and the known propagator $U_{H_{0}}\left(t, t_{0}\right)$. We constructed, with the help of $T_{1}(t)$, a new Hamiltonian $H_{2}(t)=H_{1}^{\mathrm{e}}(t)+\epsilon^{2} V_{2}(t)$ and its propagator $U_{H_{2}}\left(t, t_{0}\right)$. We approximated these operators to first order by retaining only the effective Hamiltonian $H_{1}^{\mathrm{e}}(t)$ and its propagator $U_{H_{1}^{\mathrm{e}}}\left(t, t_{0}\right)$, discarding thus the remainder $\epsilon^{2} V_{2}(t)$ and the related propagator $U_{R_{2}}\left(t, t_{0} ; t_{1}^{\prime \prime}\right)$.

To go one step further, unlike standard perturbation theory which reduces the size of the remainder from $\epsilon^{2}$ to $\epsilon^{3}$, the KAM algorithm takes advantage of the fact that after one iteration it produces a new perturbation $\epsilon^{2} V_{2}(t)$ whose order is the square of that of the original perturbation $\epsilon V_{1}(t)$. Hence by considering $H_{2}(t)$ and in particular the perturbation $\epsilon^{2} V_{2}(t)$ as the new starting point, a KAM transformation $T_{2}(t)$ produces a new perturbation $\epsilon^{4} V_{3}(t)$ [whose order is indeed $\left(\epsilon^{2}\right)^{2}$ ]. This is the essence of the superexponential character of the KAM algorithm. The fact that the new perturbation is of order $\epsilon^{4}$ instead of $\epsilon^{3}$ as in a standard perturbation theory, allows one to anticipate the importance of keeping higher order terms in $\epsilon^{2} V_{2}(t)$, in particular terms of order $\epsilon^{3}$ which would otherwise be absent if one further iterates the algorithm.

The second KAM iteration amounts thus to reproduce the first iteration on the newly constructed Hamiltonian $H_{2}(t)=H_{1}^{\mathrm{e}}(t)+\epsilon^{2} V_{2}(t)$ : the effective Hamiltonian $H_{1}^{\mathrm{e}}(t)$ and propagator $U_{H_{1}^{\mathrm{e}}}\left(t, t_{0}\right)$ now play the role of the previous unperturbed Hamiltonian and propagator respectively, we replace $\epsilon$ by $\epsilon^{2}$ and increase each subscript by one unit. The perturbation $\epsilon^{2} V_{2}(t)$ is given by Eq. (26) which we expand using Eqs. (27)-(28) and the Hausdorff formula [27, 28]

$$
\begin{aligned}
e^{A} B e^{-A} & =B+\frac{1}{1 !}[A, B]+\frac{1}{2 !}[A,[A, B]] \\
& +\frac{1}{3 !}[A,[A,[A, B]]]+\cdots,
\end{aligned}
$$

to obtain

$$
\begin{aligned}
\epsilon^{2} V_{2} & =\frac{i \epsilon^{2}}{2}\left[W_{1}, V_{1}+D_{1}\right]-\frac{\epsilon^{3}}{6}\left[W_{1},\left[W_{1}, 2 V_{1}+D_{1}\right]\right] \\
& -\frac{i \epsilon^{4}}{24}\left[W_{1},\left[W_{1},\left[W_{1}, 3 V_{1}+D_{1}\right]\right]\right]+\cdots
\end{aligned}
$$

We can rewrite this expression in a compact form with the shorthand notation $\operatorname{ad}^{k}(A, B)$ for the $k$ nested commutators $[A, \cdots[A,[A, B]] \cdots]$

$$
\operatorname{ad}^{k}(A, B) \equiv \begin{cases}B & k=0 \\ {\left[A, \operatorname{ad}^{k-1}(A, B)\right]} & k \geq 1\end{cases}
$$

The perturbation we shall start from in the second iteration of the KAM algorithm reads thus

$$
\epsilon^{2} V_{2}=\sum_{k=1}^{\infty} \frac{i^{k} \epsilon^{k+1}}{(k+1) !} \operatorname{ad}^{k}\left(W_{1}, k V_{1}+D_{1}\right) \text {. }
$$

We recall that the presence of a power series in $\epsilon$ constitutes no difficulty for this algorithm. It is on the contrary crucial in this superexponential technique to keep terms up to the final order one is interested in. Terms of order $\epsilon^{3}$, for instance, do not appear through $\epsilon^{4} V_{3}(t)$ but through the second term in the series of Eq. (34) or Eq. (36) for $\epsilon^{2} V_{2}(t)$.

We now proceed to the construction of the second KAM iteration as described above. The unitary transformation $T_{2}(t)$ is such that

$$
T_{2}^{\dagger}(t) U_{H_{2}}\left(t, t_{0}\right) T_{2}\left(t_{0}\right)=U_{H_{3}}\left(t, t_{0}\right)
$$

where the propagator $U_{H_{3}}\left(t, t_{0}\right)$ is associated with the Hamiltonian

$$
H_{3}(t) \equiv H_{2}^{\mathrm{e}}(t)+\epsilon^{4} V_{3}(t)
$$

and can be expressed as

$$
U_{H_{3}}\left(t, t_{0}\right)=U_{H_{2}^{\mathrm{e}}}\left(t, t_{2}^{\prime \prime}\right) U_{R_{3}}\left(t, t_{0} ; t_{2}^{\prime \prime}\right) U_{H_{2}^{\mathrm{e}}}\left(t_{2}^{\prime \prime}, t_{0}\right) .
$$

The new effective Hamiltonian can be decomposed as

$$
H_{2}^{\mathrm{e}}(t) \equiv H_{1}^{\mathrm{e}}(t)+\epsilon^{2} D_{2}(t)
$$

and its propagator accordingly written in the form

$$
U_{H_{2}^{\mathrm{e}}}\left(t, t_{0}\right)=U_{H_{1}^{\mathrm{e}}}\left(t, t_{2}\right) U_{P_{2}}\left(t, t_{0} ; t_{2}\right) U_{H_{1}^{\mathrm{e}}}\left(t_{2}, t_{0}\right)
$$

From Eq. (2) one deduces that $U_{P_{2}}\left(t, t_{0} ; t_{2}\right)$ is associated with the Hamiltonian

$$
\epsilon^{2} P_{2}\left(t ; t_{2}\right) \equiv U_{H_{1}^{\mathrm{e}}}\left(t_{2}, t\right) D_{2}(t) U_{H_{1}^{\mathrm{e}}}\left(t, t_{2}\right)
$$

where $U_{H_{1}^{\mathrm{e}}}\left(t, t_{0}\right)$ is given by Eq. (25). The corresponding Schrödinger equation is straightforwardly integrated if $D_{2}(t)$ is taken as

$$
\begin{aligned}
D_{2}(t) & \equiv U_{H_{1}^{\mathrm{e}}}\left(t, t_{2} ; t_{1}\right) D_{2}\left(t_{2} ; t_{2}\right) U_{H_{1}^{\mathrm{e}}}\left(t_{2}, t ; t_{1}\right) \\
& \equiv D_{2}\left(t ; t_{2}\right)
\end{aligned}
$$

with $D_{2}\left(t_{2} ; t_{2}\right)$ arbitrary. Two appealing cases are

$$
\begin{aligned}
& D_{2}\left(t_{2} ; t_{2}\right) \equiv 0 \\
& D_{2}\left(t_{2} ; t_{2}\right) \equiv V_{2}\left(t_{2}\right) .
\end{aligned}
$$

One then obtains

$$
U_{P_{2}}\left(t, t_{0} ; t_{2}\right)=e^{-i\left(t-t_{0}\right) \epsilon^{2} D_{2}\left(t_{2} ; t_{2}\right)} .
$$

The new effective propagator can be rewritten using Eq. 43) as

$$
\begin{aligned}
U_{H_{2}^{\mathrm{e}}}\left(t, t_{0}\right) & \equiv U_{H_{0}}\left(t, t_{0}\right) e^{-i\left(t-t_{0}\right) \epsilon D_{1}\left(t_{0} ; t_{1}\right)} e^{-i\left(t-t_{0}\right) \epsilon^{2} D_{2}\left(t_{0} ; t_{2}\right)} \\
& \equiv U_{H_{2}^{\mathrm{e}}}\left(t, t_{0} ; t_{1}, t_{2}\right)
\end{aligned}
$$

We now come to the definition of $T_{2}(t)$ given in Eq. 37 and require that $\epsilon^{4} V_{3}(t)$ contain no term of order lower 
than $\epsilon^{4}$. Inserting $T_{2}(t)$ into the Schrödinger equation for $U_{H_{3}}\left(t, t_{0}\right)$ one arrives at

$\epsilon^{4} V_{3}(t)=T_{2}^{\dagger}(t) H_{2}(t) T_{2}(t)-H_{2}^{\mathrm{e}}(t)-T_{2}^{\dagger}(t) i \frac{\partial}{\partial t} T_{2}(t)$.

We write

$$
T_{2}(t) \equiv e^{-i \epsilon^{2} W_{2}(t)},
$$

where $W_{2}(t)$ is allowed to depend on $\epsilon$ (although we do not indicate it explicitly). Substituting into Eq. (47) and requiring that all terms of order lower than $\epsilon^{4}$ vanish identically leads to the differential equation

$$
\frac{\partial}{\partial t} W_{2}(t)=V_{2}(t)-D_{2}(t)+i\left[W_{2}(t), H_{1}^{\mathrm{e}}(t)\right] .
$$

Its general solution reads

$$
\begin{aligned}
W_{2}(t) & =\int_{t_{2}^{\prime}}^{t} d u U_{H_{1}^{\mathrm{e}}}(t, u)\left[V_{2}(u)-D_{2}\left(u ; t_{2}\right)\right] U_{H_{1}^{\mathrm{e}}}(u, t) \\
& +U_{H_{1}^{\mathrm{e}}}\left(t, t_{0}\right) B_{2} U_{H_{1}^{\mathrm{e}}}\left(t_{0}, t\right),
\end{aligned}
$$

where $B_{2}$ is any constant self-adjoint operator. Here we shall set $B_{2}$ to 0 .

The propagator $U_{H_{1}}\left(t, t_{0}\right)$ we are looking for is obtained from Eqs. (15) and (37)

$$
U_{H_{1}}\left(t, t_{0}\right)=T_{1}(t) T_{2}(t) U_{H_{3}}\left(t, t_{0}\right) T_{2}^{\dagger}\left(t_{0}\right) T_{1}^{\dagger}\left(t_{0}\right) .
$$

Substituting Eq. (39) and neglecting $U_{R_{3}}\left(t, t_{0} ; t_{2}^{\prime \prime}\right)$ since it is associated with a Hamiltonian of order $\epsilon^{4}$, we find

$$
U_{H_{1}}\left(t, t_{0}\right)=U_{H_{1}}^{(2)}\left(t, t_{0}\right)+\mathcal{O}\left(\epsilon^{4}\right),
$$

with the two-iteration KAM expansion

$$
U_{H_{1}}^{(2)}\left(t, t_{0}\right)=T_{1}(t) T_{2}(t) U_{H_{2}^{\mathrm{e}}}\left(t, t_{0}\right) T_{2}^{\dagger}\left(t_{0}\right) T_{1}^{\dagger}\left(t_{0}\right) .
$$

This expansion features two additional arbitrary times $\left(t_{2}\right.$ and $\left.t_{2}^{\prime}\right)$ and two additional arbitrary operators $\left(D_{2}\left(t_{2} ; t_{2}\right)\right.$ and $\left.B_{2}\right)$ that can be chosen so as to improve its accuracy. The structure of the equations and of the operators involved is exactly the same for the second KAM iteration as for the first one, and will be the same for any iteration. In particular, the effective perturbation determined at each step is always of the form of Eq. (36) since each step is just a renaming of the previous one. This is in contrast to the order by order methods where the determination of that operator requires each time more algebra.

\section{Summary of the time-dependent KAM algorithm}

The propagator of the perturbed Hamiltonian $H_{1}(t)=$ $H_{0}(t)+\epsilon V_{1}(t)$ is approximated after $n$ iterations according to

$$
U_{H_{1}}\left(t, t_{0}\right)=U_{H_{1}}^{(n)}\left(t, t_{0}\right)+\mathcal{O}\left(\epsilon^{2^{n}}\right),
$$

with the unitary $n$-iteration KAM expansion

$$
\begin{aligned}
U_{H_{1}}^{(n)}\left(t, t_{0}\right) & =T_{1}(t) \ldots T_{n}(t) U_{H_{n}^{\mathrm{e}}}\left(t, t_{0}\right) \\
& \times T_{n}^{\dagger}\left(t_{0}\right) \ldots T_{1}^{\dagger}\left(t_{0}\right) .
\end{aligned}
$$

We recall that the superexponential character of the correction terms stems from the fact that each KAM transformation reduces the perturbation to a new effective perturbation whose order is squared. One defines

$$
\begin{aligned}
& U_{H_{n}^{\mathrm{e}}}\left(t, t_{0}\right) \equiv U_{H_{0}}\left(t, t_{0}\right) \exp \left[-i\left(t-t_{0}\right) \epsilon D_{1}\left(t_{0} ; t_{1}\right)\right] \\
& \times \cdots \times \exp \left[-i\left(t-t_{0}\right) \epsilon^{2^{n-1}} D_{n}\left(t_{0} ; t_{n}\right)\right]
\end{aligned}
$$

with the possibility to choose one of the following for any $k \geq 1$

$$
\begin{aligned}
D_{k}\left(t ; t_{k}\right) & \equiv 0 \\
D_{k}\left(t ; t_{k}\right) & \equiv U_{H_{k-1}^{\mathrm{e}}}\left(t, t_{k}\right) V_{k}\left(t_{k}\right) U_{H_{k-1}^{\mathrm{e}}}\left(t_{k}, t\right) .
\end{aligned}
$$

Each KAM transformation reads

$$
T_{k}(t) \equiv \exp \left[-i \epsilon^{2^{k-1}} W_{k}(t)\right],
$$

where

$$
W_{k}(t) \equiv \int_{t_{k}^{\prime}}^{t} d u U_{H_{k-1}^{\mathrm{e}}}(t, u)\left[V_{k}(u)-D_{k}\left(u ; t_{k}\right)\right] U_{H_{k-1}^{\mathrm{e}}}(u, t) .
$$

One has $U_{H_{0}^{\mathrm{e}}}\left(t, t_{0}\right) \equiv U_{H_{0}}\left(t, t_{0}\right)$. To continue the algorithm we determine the new perturbation $\epsilon^{2^{n}} V_{n+1}(t)$ in terms of the operators $W_{n}(t), V_{n}(t)$ and $D_{n}\left(t ; t_{n}\right)$ obtained at the preceding step

$$
V_{n+1} \equiv \sum_{k=1}^{\infty} \frac{i^{k}\left(\epsilon^{2^{n-1}}\right)^{k-1}}{(k+1) !} \operatorname{ad}^{k}\left(W_{n}, k V_{n}+D_{n}\right),
$$

with the usual definition of $\operatorname{ad}(A, B)$ recalled in Eq. (35) and where the infinite series can be truncated to a prescribed order. This new effective perturbation has exactly the same structure at each iteration which is useful for applications, particularly when high orders computations are needed.

At each KAM iteration two arbitrary times $t_{k}$ and $t_{k}^{\prime}$ are introduced through Eqs. (57) and (59). As a consequence, one has the following dependence on these free parameters:

$$
\begin{aligned}
U_{H_{n}^{\mathrm{e}}}\left(t, t_{0}\right) & \equiv U_{H_{n}^{\mathrm{e}}}\left(t, t_{0} ; t_{1}, \cdots, t_{n}\right), \\
T_{n}(t) & \equiv T_{n}\left(t ; t_{1}, \cdots, t_{n}, t_{1}^{\prime}, \cdots, t_{n}^{\prime}\right), \\
W_{n}(t) & \equiv W_{n}\left(t ; t_{1}, \cdots, t_{n}, t_{1}^{\prime}, \cdots, t_{n}^{\prime}\right), \\
V_{n}(t) & \equiv V_{n}\left(t ; t_{1}, \cdots, t_{n-1}, t_{1}^{\prime}, \cdots, t_{n-1}^{\prime}\right) .
\end{aligned}
$$

These quantities, together with the choice of Eq. (57a or (57b) for the arbitrary operator $D_{n}\left(t_{n} ; t_{n}\right)$, may significantly affect the accuracy of the $n$-iteration KAM expansion. 


\section{Comparison with the Magnus expansion}

The Magnus and KAM expansions differ in several respects. First it is remarkable that the KAM algorithm can be implemented in the original representation. In Appendix [C] we show that the result obtained for the KAM expansion in the interaction representation is identical at any level of approximation.

Most importantly of course is the superexponential character of the KAM expansion which manifests itself as of the second iteration. However, the first iteration of these algorithms are generally different, owing to the non-commutativity of the operators involved. Indeed, Eq. (12) gives for the first order Magnus expansion

$$
U_{H_{1}}^{(1)}\left(t, t_{0}\right)=e^{-i \epsilon M_{1}\left(t ; t_{0}\right)} U_{H_{0}}\left(t, t_{0}\right),
$$

while the one-iteration KAM expansion of Eq. (31) reads

$$
\begin{aligned}
U_{H_{1}}^{(1)}\left(t, t_{0}\right) & =e^{-i \epsilon\left\{M_{1}\left(t ; t_{0}\right)-M_{1}\left(t_{1}^{\prime} ; t_{0}\right)+\left(t_{1}^{\prime}-t\right) D_{1}\left(t ; t_{1}\right)\right\}} \\
& \times e^{-i \epsilon\left(t-t_{0}\right) D_{1}\left(t ; t_{1}\right)} e^{-i \epsilon\left\{M_{1}\left(t_{1}^{\prime} ; t_{0}\right)+\left(t_{0}-t_{1}^{\prime}\right) D_{1}\left(t ; t_{1}\right)\right\}} \\
& \times U_{H_{0}}\left(t, t_{0}\right) .
\end{aligned}
$$

We recall from Eq. (13) that

$$
M_{1}\left(t ; t_{0}\right)=\int_{t_{0}}^{t} d u U_{H_{0}}(t, u) V_{1}(u) U_{H_{0}}(u, t) .
$$

To compare these expressions we shall cast the product of the exponentials of Eq. (63) into a single exponential using the Campbell-Baker-Hausdorff formula [23, 27]

$$
e^{A} e^{B}=e^{C},
$$

where

$$
C=A+B+\frac{1}{2}[A, B]+\frac{1}{12}[A-B,[A, B]]+\cdots .
$$

We note that the exponents of Eq. (63) precisely sum up to that of Eq. (62), i.e., $\epsilon M_{1}(t)$. Hence, by Eq. 65b), these expansions differ by terms of order $\epsilon^{2}$. In other words, these expansions differ through terms whose order is that of their remainder, which therefore enables one to recover precisely the same expansion up to a given order. To compute explicitly these terms of order $\epsilon^{2}$ and show that they generally do not vanish we apply the CampbellBaker-Hausdorff formula twice to reduce the three exponentials of Eq. (63) to a single one

$$
\begin{aligned}
U_{H_{1}}^{(1)}\left(t, t_{0}\right) & =e^{-i \epsilon M_{1}\left(t ; t_{0}\right)-\frac{\epsilon^{2}}{2} K_{1}\left(t ; t_{0}, t_{1}, t_{1}^{\prime}\right)+O\left(\epsilon^{3}\right)} \\
& \times U_{H_{0}}\left(t, t_{0}\right),
\end{aligned}
$$

where

$$
\begin{aligned}
K_{1}\left(t ; t_{0}, t_{1}, t_{1}^{\prime}\right) & \equiv\left(t-t_{1}^{\prime}\right)\left[M_{1}\left(t ; t_{0}\right), D_{1}\left(t ; t_{1}\right)\right] \\
& +\left(t_{0}-t\right)\left[M_{1}\left(t_{1}^{\prime} ; t_{0}\right), D_{1}\left(t ; t_{1}\right)\right] \\
& +\left[M_{1}\left(t ; t_{0}\right), M_{1}\left(t_{1}^{\prime} ; t_{0}\right)\right] .
\end{aligned}
$$

We recall that, according to Eq. (23), we choose either $D_{1}\left(t ; t_{1}\right)=0$ or $D_{1}\left(t ; t_{1}\right)=U_{H_{0}}\left(t, t_{1}\right) V_{1}\left(t_{1}\right) U_{H_{0}}\left(t_{1}, t\right)$. As a consequence $K_{1}\left(t ; t_{0}, t_{1}, t_{1}^{\prime}\right)$ is generally non zero and therefore, at this first level of approximation, the KAM and Magnus techniques differ by terms of order $\epsilon^{2}$. However, if one chooses $D_{1}\left(t ; t_{1}\right)=0$ together with $t_{1}^{\prime}=t_{0}$, then the one-iteration KAM expansion and the first order Magnus expansion coincide (this will no longer be true for the next levels of approximation).

Note that for the KAM algorithm, as we discuss in the following section, the choice of $D_{1}\left(t ; t_{1}\right)$ with an arbitrary $t_{1}$ allows one to enhance the convergence precisely by acting on these higher order terms (we emphasize that these terms appear here as higher order ones because of the use of the Campbell-Baker-Hausdorff formula; but at the level of Eq. (63), each exponent is indeed of order $\epsilon$ ).

For higher orders and iterations however the KAM algorithm is a priori expected to perform far better owing to both the superexponential character and the possibility to enhance the accuracy.

\section{IMPROVING THE ACCURACY}

The formulation of the time-dependent KAM technique presented in Sec. ID reveals the existence of several degrees of freedom which are at our disposal to reduce the error without prior knowledge of the exact solution: i) the choice of Eq. (57) for the operators $D_{k}\left(t ; t_{k}\right)$, and ii) the free parameters $t_{k}$ and $t_{k}^{\prime}$ of Eqs. (57b) and (59). There is also a third way discussed below: iii) the possibility to consider another identification of the perturbation and unperturbed Hamiltonian.

The items i) and ii) also apply to the Poincaré-Von Zeipel and the Van Vleck techniques, although to a smaller extent, as we describe below.

\section{A. Choice of $D_{k}\left(t ; t_{k}\right)$ and correspondence between resonances and secular terms.}

Each iteration of the time-dependent Poincaré-Von Zeipel, the time-dependent Van Vleck and the timedependent KAM algorithms features an arbitrary operator $D_{k}\left(t_{k} ; t_{k}\right)$. In the preceding section, in addition to the simplest case $D_{k}\left(t_{k} ; t_{k}\right)=0$, we suggested the choice $D_{k}\left(t_{k} ; t_{k}\right)=V_{k}\left(t_{k}\right)$ where $t_{k}$ is an arbitrary time.

The first iteration involves the operator $D_{1}\left(t ; t_{1}\right)$ which satisfies the same equation in the three algorithms, namely Eq. (22). This equation is actually the general solution to the differential equation

$$
\left[H_{0}(t), D_{1}\left(t ; t_{1}\right)\right]=i \frac{\partial}{\partial t} D_{1}\left(t ; t_{1}\right)
$$

This latter equation, together with Eq. (28) for $W_{1}(t)$, are the time-dependent generalization of the so-called cohomology equations [29] considered in the stationary case. 
The time-independent problem, i.e. the problem of finding a transformation $T_{1}$ that enables one to simplify the time-independent Hamiltonian $H_{1}$ according to $T_{1}^{\dagger} H_{1} T_{1}=H_{0}+\epsilon D_{1}+\epsilon^{2} V_{2}$, is recovered when one conveniently chooses $T_{1}$ as time-independent. This transformation is sometimes called contact transformation 32] or level-shift transformation [28].

In this case all the operators, and in particular $D_{1}$ and $W_{1}$, are time-independent and the standard cohomology equations are recovered

$$
\begin{aligned}
& {\left[H_{0}, D_{1}\right]=0,} \\
& V_{1}-D_{1}+i\left[W_{1}, H_{0}\right]=0 .
\end{aligned}
$$

Their solutions can be determined using the following key property [29]: $W_{1}$ exists if and only if $\Pi_{H_{0}}\left(D_{1}-\right.$ $\left.V_{1}\right)=0$, where $\Pi_{H_{0}}$ is the projector in the kernel of the application $A \mapsto\left[A, H_{0}\right]$ (for an operator $A$ acting on the same Hilbert space as $\left.H_{0}\right)$. The projector $\Pi_{H_{0}}$ applied on an operator $A$ captures thus all the part $B$ of $A$ which commutes with $H_{0}$ : $\left[B, H_{0}\right]=0$. The unique solution $D_{1}$ allowing $W_{1}$ to exist and satisfying Eq. (68) is thus

$$
D_{1}=\Pi_{H_{0}} V_{1} \equiv \lim _{T \rightarrow \infty} \frac{1}{T} \int_{0}^{T} e^{-i t H_{0}} V_{1} e^{i t H_{0}} .
$$

The resonances are associated with terms of $V_{1}$ which commute with $H_{0}$. Application of Eq. (70) can be interpreted as an averaging of $V_{1}$ with respect to $H_{0}$ which allows one to extract resonances.

For the time-dependent problem, the general solution to Eq. (28) is given by Eq. (29). Defining the average

$$
\Pi_{-} V_{1} \equiv \lim _{\tau \rightarrow \infty} \frac{1}{\tau} \int_{t-\tau}^{t} d s U_{H_{0}}(t, s) V_{1}(s) U_{H_{0}}(s, t),
$$

one can show the following property: if $W_{1}(t)$ is bounded for negative infinite times, then $\Pi_{-}\left(V_{1}-D_{1}\right)=0$. This is satisfied by $D_{1}=\Pi_{-} V_{1}$, the only solution compatible with Eq. (68) and the projector $\Pi_{-}$. This means that the averaging $D_{1}=\Pi_{-} V_{1}$ allows to remove secular terms at negative infinite times. We remark that this definition of the average, Eq. (71), can in fact be recovered from the formal calculation of the average $\Pi_{K_{0}} V_{1}$ of Eq. (70) with respect to $K_{0}=-i \frac{\partial}{\partial t}+H_{0}$ in an extended space, which includes time as a coordinate [14, 20]. This gives the precise correspondence between the resonances of stationary problems and the secular terms of timeindependent problems.

In Ref. 14] it was shown for perturbations that are localized in time, in a finite but possibly large interval $t_{i} \leq t \leq t_{f}$, that Eq. (71) reduces to

$$
\Pi_{-} V_{1}=U_{H_{0}}\left(t, t_{i}\right) V_{1}\left(t_{i}\right) U_{H_{0}}\left(t_{i}, t\right) .
$$

This is a particular solution to Eq. (68) corresponding to the choice $t_{1} \equiv t_{i}$ in Eqs. (22) and (23b). An alternate definition of the average

$$
\Pi_{+} V_{1} \equiv \lim _{\tau \rightarrow \infty} \frac{1}{\tau} \int_{t}^{t+\tau} d s U_{H_{0}}(t, s) V_{1}(s) U_{H_{0}}(s, t),
$$

gives a different result

$$
\Pi_{+} V_{1}=U_{H_{0}}\left(t, t_{f}\right) V_{1}\left(t_{f}\right) U_{H_{0}}\left(t_{f}, t\right),
$$

and allows one to remove secular terms at positive infinite times. Generally one cannot remove simultaneously the secular terms at negative and positive large times. This shows a conceptual difference between stationary resonances and secular terms associated with perturbations localized in time. Furthermore, it suggests that combining both definitions in a non-trivial way gives a new secular term that could improve the convergence of the algorithm. This is achieved by the general solution to Eq. (68), Eq. (22), written with the perturbation evaluated at a free time $t_{1}$ as the arbitrary operator [cf. Eq. 23b]

$$
D_{1}\left(t ; t_{1}\right) \equiv U_{H_{0}}\left(t, t_{1}\right) V_{1}\left(t_{1}\right) U_{H_{0}}\left(t_{1}, t\right) .
$$

The free $t_{1}$ can then be chosen as we describe below to minimize the remainder obtained at the first iteration of the perturbative algorithm.

The next iterations also offer the possibility to choose for $D_{k}\left(t ; t_{k}\right)$, in particular 0 or $U_{H_{k-1}^{\mathrm{e}}}\left(t, t_{k}\right) V_{k}\left(t_{k}\right) U_{H_{k-1}^{\mathrm{e}}}\left(t_{k}, t\right)$ similarly to Eq. (75). Note however that for the Poincaré-Von Zeipel and the Van Vleck techniques, the choice corresponding to $V_{k}\left(t_{k}\right)$ can only be made once (for an arbitrary value of $k$ ). As explained in Appendixes A and B, respectively, this is due to the order by order character of these techniques.

\section{B. Enhancing the convergence}

After one iteration of the KAM algorithm one deduces from Eqs. (15) and (17) an exact expression for the full propagator

$$
\begin{aligned}
U_{H_{1}}\left(t, t_{0}\right) & =T_{1}\left(t ; t_{1}, t_{1}^{\prime}\right) U_{H_{1}^{\mathrm{e}}}\left(t, t_{1}^{\prime \prime} ; t_{1}\right) U_{R_{2}}\left(t, t_{0} ; t_{1}, t_{1}^{\prime}, t_{1}^{\prime \prime}\right) \\
& \times U_{H_{1}^{\mathrm{e}}}\left(t_{1}^{\prime \prime}, t_{0} ; t_{1}\right) T_{1}^{\dagger}\left(t_{0} ; t_{1}, t_{1}^{\prime}\right),
\end{aligned}
$$

where $t_{1}, t_{1}^{\prime}$ and $t_{1}^{\prime \prime}$ are arbitrary times [cf. Eq. [321]. The propagator $U_{R_{2}}\left(t, t_{0} ; t_{1}, t_{1}^{\prime}, t_{1}^{\prime \prime}\right)$ of the Hamiltonian given in Eq. (18) is associated with a second order generator

$$
U_{R_{2}}\left(t, t_{0} ; t_{1}, t_{1}^{\prime}, t_{1}^{\prime \prime}\right) \equiv e^{-i \epsilon^{2} G_{2}\left(t ; t_{0}, t_{1}, t_{1}^{\prime}, t_{1}^{\prime \prime}\right)} .
$$

To obtain the one-iteration KAM expansion $U_{H_{1}}^{(1)}\left(t, t_{0}\right)$ we neglected this propagator, replacing it by the identity in the above product. Obviously, the closer $U_{R_{2}}\left(t, t_{0} ; t_{1}, t_{1}^{\prime}, t_{1}^{\prime \prime}\right)$ is to the identity, the smaller the correction terms are, i.e., the more accurate the approximation is. We can improve this accuracy if we can make that propagator closer to the identity, or equivalently, its generator closer to zero. The distance is defined through the norm $\|A\|=\max _{\|\psi\|=1}\|A \psi\|$ with $\psi$ in the Hilbert space of the problem. For an Hermitian matrix, this norm is the largest absolute value of its eigenvalues. 
We calculate this generator $\epsilon^{2} G_{2}\left(t ; t_{0}, t_{1}, t_{1}^{\prime}, t_{1}^{\prime \prime}\right)$ by solving the Schrödinger equation with the Hamiltonian of Eq. (18) in the form of an exponential using Eq. (66). This is a time-dependent problem with a zero unperturbed Hamiltonian and whose perturbation is $\epsilon^{2} U_{H_{1}^{\mathrm{e}}}\left(t_{1}^{\prime \prime}, t ; t_{1}\right) V_{2}\left(t ; t_{1}, t_{1}^{\prime}\right) U_{H_{1}^{\mathrm{e}}}\left(t, t_{1}^{\prime \prime} ; t_{1}\right)$. Hence, we evaluate the lowest order contribution to $G_{2}\left(t ; t_{0}, t_{1}, t_{1}^{\prime}, t_{1}^{\prime \prime}\right)$ as

$$
\begin{aligned}
G_{2}^{(2)}\left(t ; t_{0}, t_{1}, t_{1}^{\prime}\right) \equiv \int_{t_{0}}^{t} d u & U_{H_{1}^{\mathrm{e}}}\left(t_{0}, u ; t_{1}\right) V_{2}\left(u ; t_{1}, t_{1}^{\prime}\right) \\
\times & U_{H_{1}^{\mathrm{e}}}\left(u, t_{0} ; t_{1}\right)
\end{aligned}
$$

where we set $t_{1}^{\prime \prime}=t_{0}$ (its precise value is not relevant since the one-iteration KAM expansion $U_{H_{1}}^{(1)}\left(t, t_{0} ; t_{1}, t_{1}^{\prime}\right)$ is independent of the parameter $\left.t_{1}^{\prime \prime}\right)$. It is this operator $G_{2}^{(2)}\left(t ; t_{0}, t_{1}, t_{1}^{\prime}\right)$ that has to remain small for the algorithm to converge [35]. Having the arbitrary times $t_{1}$ and $t_{1}^{\prime}$ at our disposal, we can actually enhance the convergence of the algorithm by minimizing the norm of this operator with respect to these free parameters.

Similarly, the $n$-iteration KAM expansion of Eq. (55) can be optimized by minimizing the norm of the following operator with respect to one or several of the free parameters $t_{1}, \cdots, t_{n}, t_{1}^{\prime}, \cdots, t_{n}^{\prime}$

$$
\begin{aligned}
G_{n+1}^{(n+1)}(t) & \equiv \int_{t_{0}}^{t} d u U_{H_{n}^{\mathrm{e}}}\left(t_{0}, u\right) V_{n+1}(u) U_{H_{n}^{\mathrm{e}}}\left(u, t_{0}\right) \\
& \equiv G_{n+1}^{(n+1)}\left(t ; t_{1}, \cdots, t_{n}, t_{1}^{\prime}, \cdots, t_{n}^{\prime}\right) .
\end{aligned}
$$

The dependence of $U_{H_{n}^{\mathrm{e}}}\left(t, t_{0}\right)$ and $V_{n+1}(t)$ on these parameters is given in Eq. 661.

It turns out, as will be illustrated in Sec. IVB that modifying the parameters $t_{k}$ and/or $t_{k}^{\prime}$ can improve the accuracy by more than one order of magnitude already for $k=1$.

\section{KAM expansion with another identification of the unperturbed Hamiltonian}

The perturbed Hamiltonian can be written as

$$
\begin{aligned}
& H_{1}(t)=\underbrace{H_{0}(t)+\epsilon D_{1}\left(t ; t_{1}\right)}+\underbrace{\epsilon V_{1}(t)-\epsilon D_{1}\left(t ; t_{1}\right)} \\
& \equiv H_{0}^{\prime}\left(t ; t_{1}\right)+\epsilon V_{1}^{\prime}\left(t ; t_{1}\right),
\end{aligned}
$$

where $D_{1}\left(t ; t_{1}\right) \equiv U_{H_{0}}\left(t, t_{1}\right) V_{1}\left(t_{1}\right) U_{H_{0}}\left(t_{1}, t\right)$ with $t_{1}$ arbitrary [cf. Eqs. (22) and [23b]). The propagator associated with $H_{0}^{\prime}\left(t ; t_{1}\right)$ can always be determined since by Eqs. (19) and (25) one has

$$
\begin{aligned}
H_{0}^{\prime}\left(t ; t_{1}\right) & =H_{1}^{\mathrm{e}}\left(t ; t_{1}\right), \\
U_{H_{0}^{\prime}}\left(t, t_{0} ; t_{1}\right) & =U_{H_{1}^{\mathrm{e}}}\left(t, t_{0} ; t_{1}\right) .
\end{aligned}
$$

We now apply the KAM algorithm exactly as summarized in Sec. IID 3 i.e. with the same definitions for all the operators involved in the expansion, but with the identification of Eq. (80). This decomposition has the property that $V_{1}^{\prime}\left(t_{1} ; t_{1}\right)=0$ which implies by Eq. 23b that $D_{1}^{\prime}\left(t ; t_{1}\right)=0$. The free parameter $t_{1}$ is therefore introduced here through Eq. (81b). One arrives at a KAM expansion which is still of the form of Eq. (55) but may significantly differ from that resulting from the conventional decomposition.

We emphasize that the possibility to consider the identification of Eq. (80) as a new starting point for a perturbative treatment is specific to the KAM technique which is not an order by order method, contrary to the Magnus, the Poincaré-Von Zeipel and the Van Vleck algorithms.

\section{BEYOND THE SUDDEN APPROXIMATION}

\section{A. Preliminaries}

We consider a system described by the Hamiltonian $\mathrm{H}$ (autonomous or not). It is perturbed by a timedependent Hamiltonian $\mathrm{V}(\mathrm{t})$ whose characteristic duration is $\tau$. This latter quantity is the time during which the interaction differs significantly from zero, and not the full duration of the interaction, which may be large but finite. Here we define $\tau$ as twice the full width at half maximum, having in mind a perturbation which presents a time-dependent envelope. We assume that the perturbation $\mathrm{V}(\mathrm{t})$ satisfies

$$
\left[\mathrm{V}(\mathrm{t}), \mathrm{V}\left(\mathrm{t}_{0}\right)\right]=0 \quad \forall \mathrm{t}, \mathrm{t}_{0},
$$

which is realized in many situations of physical interest. The propagator of the perturbed system evolves according to the Schrödinger equation

$$
i \hbar \frac{\partial}{\partial \mathrm{t}} \mathrm{U}\left(\mathrm{t}, \mathrm{t}_{0}\right)=\{\mathrm{H}+\mathrm{V}(\mathrm{t})\} \mathrm{U}\left(\mathrm{t}, \mathrm{t}_{0}\right),
$$

with $\mathrm{U}\left(\mathrm{t}_{0}, \mathrm{t}_{0}\right)=\mathbb{1}$, the identity operator on the appropriate Hilbert space $\mathbb{H}$. We define a dimensionless time $t$ and dimensionless operators $H, V(t)$ and $U\left(t, t_{0}\right)$ through

$$
\begin{aligned}
\mathrm{t} & \equiv \tau t, \\
\mathrm{H} & \equiv \hbar \omega H, \\
\mathrm{~V}(\mathrm{t}) & \equiv \frac{\hbar}{\tau} V(t), \\
\mathrm{U}\left(\mathrm{t}, \mathrm{t}_{0}\right) & \equiv U\left(t, t_{0}\right),
\end{aligned}
$$

where $\omega$ is some characteristic frequency of $\boldsymbol{H}$. In dimensionless units Eq. (83) becomes

$$
i \frac{\partial}{\partial t} U\left(t, t_{0}\right)=\{V(t)+\epsilon H\} U\left(t, t_{0}\right),
$$

where we define a sudden parameter $\epsilon \equiv \omega \tau$.

The sudden or impulsive regime corresponds to the limit $\epsilon \rightarrow 0$. Our aim is to obtain a perturbative expansion for the evolution operator $U\left(t, t_{0}\right)$ of the perturbed Hamiltonian $V(t)+\epsilon H$ beyond the sudden regime. To 
this aim we identify the original perturbation $V(t)$ as the unperturbed Hamiltonian $H_{0}(t)$ and the original unperturbed Hamiltonian $H$ as the perturbation $V_{1}$

$$
\begin{aligned}
H_{0}(t) & \equiv V(t), \\
V_{1} & \equiv H .
\end{aligned}
$$

Note that we need only consider a finite interval of time as $V(t)$ is localized in time. By virtue of Eq. (82), the propagator for $\epsilon=0$ reads

$$
U_{H_{0}}\left(t, t_{0}\right)=\exp \left\{-i \int_{t_{0}}^{t} V(u) d u\right\} .
$$

We shall follow this approach below and consider the various perturbative schemes described in Sec. III in the case of two-level systems driven by short pulses.

\section{B. Illustration on pulse-driven two-level systems}

Our purpose is to compare the various algorithms and investigate the convergence enhancement as well as to show that the unitary time-dependent KAM theory is well suited to study regimes beyond the impulsive or sudden limit.

In the notations of the preliminaries, we take $\mathrm{H}=\hbar \omega \sigma_{3}$ and $\mathrm{V}(\mathrm{t})=\frac{\hbar}{\tau} \Omega(t) \sigma_{1}$ where $\Omega(t)$ is a pulse shape function and $\sigma_{k}$ are the Pauli matrices

$$
\sigma_{1}=\left(\begin{array}{cc}
0 & 1 \\
1 & 0
\end{array}\right), \quad \sigma_{2}=\left(\begin{array}{cc}
0 & -i \\
i & 0
\end{array}\right), \quad \sigma_{3}=\left(\begin{array}{cc}
1 & 0 \\
0 & -1
\end{array}\right) .
$$

Hence, the operators defined in Eq. (86) are here $H_{0}(t)=$ $\Omega(t) \sigma_{1}$ and $V_{1}=\sigma_{3}$. The Schrödinger equation reads

$$
i \frac{\partial}{\partial t} U_{H_{1}}\left(t, t_{0}\right)=\left[\Omega(t) \sigma_{1}+\epsilon \sigma_{3}\right] U_{H_{1}}\left(t, t_{0}\right),
$$

with $U_{H_{1}}\left(t_{0}, t_{0}\right)=\mathbb{1}_{\mathbb{C}^{2}}$. For $\epsilon=0$ its solution is

$$
U_{H_{0}}\left(t, t_{0}\right) \equiv e^{-i A(t) \sigma_{1}},
$$

where $A(t) \equiv \int_{t_{0}}^{t} \Omega(u) d u$. The pulse area $A \equiv A(\infty)$ is a dimensionless parameter that can be fixed independently of the sudden parameter $\epsilon(\equiv \omega \tau)$ that we take here as the perturbative parameter. This allows us, in particular, to treat large nonperturbative areas for short pulse durations.

The Magnus expansion $U_{H_{1}}^{(n)}\left(t, t_{0}\right)$ is given by Eqs. (12)(14a).

The KAM expansion is obtained from Eqs. (55)-(60). We distinguish three types of KAM expansions, reflecting the choices discussed in Secs. IID III and IIC]

Type A: each iteration $k$ involves the operator $D_{k}\left(t_{k} ; t_{k}\right)=0$ [cf. Eq. (157a)].

Type B: $D_{k}\left(t_{k} ; t_{k}\right)=V_{k}\left(t_{k}\right)$ for all $k$ [cf. Eq. [57b)].
Type C: the unperturbed Hamiltonian is defined as $H_{0}^{\prime}\left(t ; t_{1}\right)=H_{0}(t)+\epsilon U_{H_{0}}\left(t, t_{1}\right) V_{1}\left(t_{1}\right) U_{H_{0}}\left(t_{1}, t\right)[\mathrm{cf}$. Eq. [80)].

For the type A $n$-iteration expansion one has $n$ free times

$$
U_{H_{1}}^{(n)}\left(t, t_{0}\right)=U_{H_{1}}^{(n)}\left(t, t_{0} ; t_{1}^{\prime}, \cdots, t_{n}^{\prime}\right),
$$

while for the types $\mathrm{B}$ and $\mathrm{C}$ one has $2 n$ such parameters

$$
U_{H_{1}}^{(n)}\left(t, t_{0}\right)=U_{H_{1}}^{(n)}\left(t, t_{0} ; t_{1}, \cdots, t_{n}, t_{1}^{\prime}, \cdots, t_{n}^{\prime}\right) .
$$

In the case of two-level systems, the infinite series of Eq. (60) for the new effective KAM perturbation $V_{n+1}(t)$ can be cast into the form [14]

$$
\begin{aligned}
V_{n+1} & =\left[W_{n}, a_{n} V_{n}+b_{n} D_{n}\right] \\
& +\epsilon_{n}\left[W_{n},\left[W_{n}, c_{n} V_{n}+d_{n} D_{n}\right]\right],
\end{aligned}
$$

where

$$
\begin{aligned}
& a_{n} \equiv i \frac{\cos \epsilon_{n} \lambda_{n}+\epsilon_{n} \lambda_{n} \sin \epsilon_{n} \lambda_{n}-1}{\epsilon_{n}^{2} \lambda_{n}^{2}}, \quad b_{n} \equiv i \frac{1-\cos \epsilon_{n} \lambda_{n}}{\epsilon_{n}^{2} \lambda_{n}^{2}}, \\
& c_{n} \equiv \frac{\epsilon_{n} \lambda_{n} \cos \epsilon_{n} \lambda_{n}-\sin \epsilon_{n} \lambda_{n}}{\epsilon_{n}^{3} \lambda_{n}^{3}}, \quad d_{n} \equiv c_{n}+i b_{n},
\end{aligned}
$$

with $\epsilon_{n} \equiv \epsilon^{2^{n-1}}$ and $\lambda_{n}(t) \equiv \sqrt{-\operatorname{det} W_{n}(t)}$.

For given $\epsilon$ and $A$, the error $\Delta_{n}$ at the end of the pulse between the numerical solution of the Schrödinger equation and the result obtained after $n$ iterations is defined as

$$
\Delta_{n} \equiv\left\|U_{H_{1}}\left(t_{f}, t_{i}\right)-U_{H_{1}}^{(n)}\left(t_{f}, t_{i}\right)\right\| .
$$

We also define the error $\delta_{n}$ in the transition probability from the lower state $|-\rangle$ to the upper state $|+\rangle$

$$
\delta_{n} \equiv\left|\left\langle+\left|U_{H_{1}}^{(n)}\left(t_{f}, t_{i}\right)\right|-\right\rangle\right|^{2}-\left|\left\langle+\left|U_{H_{1}}\left(t_{f}, t_{i}\right)\right|-\right\rangle\right|^{2} .
$$

We consider the following dimensionless pulse shape between the dimensionless time $t_{i}=0$ and $t_{f}=1$

$$
\Omega(t)=\left\{\begin{array}{cc}
2 A \sin ^{2}(\pi t) & 0 \leq t \leq 1 \\
0 & \text { elsewhere }
\end{array}\right.
$$

Figure 1 displays the common logarithm of the error $\Delta_{1}$ and the error $\delta_{1}$ as a function of the pulse area $A$ for the Magnus expansion and the three types of oneiteration KAM expansions in the case $t_{1}=t_{1}^{\prime}=0$ and $\epsilon=0.5$. The errors $\Delta_{1}$ and $\delta_{1}$ globally decrease when $A$ increases. This is expected on the basis of Eqs. (88) and (94) as the relative importance of the perturbation then decreases. One also observes marked oscillations in $\Delta_{1}$ and $\delta_{1}$ with a pseudo-period of $\pi$. This stems from the form of the unperturbed propagator [cf. Eq. [89)] and the fact that it always appears twice, in particular in the operator $G_{2}^{(2)}\left(t ; t_{0}\right)$ of Eq. (78) which controls the error after one iteration. 
Let us recall that the one-iteration KAM expansion of type A coincides with the first order Magnus expansion for $t_{1}^{\prime}=0$. It is seen, by both measures of the error, that each of the KAM expansions can perform better than the other ones on some intervals of $A$. Hence, in order to establish a fair comparison, we shall consider the particular value $A=1$ where the first order Magnus expansion and the one-iteration KAM expansion of type $B$ yield essentially the same error $\Delta_{1}$ for $\epsilon=0.5$. This remains true for all values of $\epsilon$ up to 2 as can be seen from Fig. 2 which depicts the errors $\Delta_{1}$ and $\delta_{1}$ as a function of $\epsilon$ for $A=1$.

In Fig. 2 we also present the one-iteration KAM expansion of type $\mathrm{B}$ that is optimized by choosing $t_{1}=0.5, t_{1}^{\prime}=$ 0.22 . We see that the error $\Delta_{1}$ is reduced (with respect to the comparable Magnus and non-optimized type B KAM expansions) by more than one order of magnitude up to values of $\epsilon$ equal to unity. The error $\delta_{1}$ on the transition probability is also considerably reduced. Notice that the values of $\delta_{1}$ for the Magnus and non-optimized type B KAM expansions differ while the values of $\Delta_{1}$ are indistinguishable. For comparison, we also consider the (nonunitary) Dyson expansion [23]. Recall that it is obtained by repeated use of the integral form of the Schrödinger equation in the interaction representation with respect to $H_{0}(t)$

$$
\begin{aligned}
U_{H_{1}^{\mathrm{i}}}\left(t, t_{0} ; t_{0}\right) & =\mathbb{1}-i \int_{t_{0}}^{t} d u H_{1}^{\mathrm{i}}\left(u ; t_{0}\right) U_{H_{1}^{\mathrm{i}}}\left(u, t_{0} ; t_{0}\right) \\
& =\mathbb{1}-i \int_{t_{0}}^{t} d u H_{1}^{\mathrm{i}}\left(u ; t_{0}\right) \\
& \times\left[\mathbb{1}-i \int_{t_{0}}^{u} d v H_{1}^{\mathrm{i}}\left(v ; t_{0}\right)\right]+\cdots,
\end{aligned}
$$

where $H_{1}^{\mathrm{i}}\left(t ; t_{0}\right)$, given by Eq. (2c), contains a prefactor $\epsilon$. One then returns to the original representation with Eq. (2a). For the value of $A$ considered in Fig. 2, the Dyson expansion yields the largest error $\Delta_{1}$ whereas its error on the transition probability is rather small.

In Fig. 3 we plot $g_{2}$ defined as the largest absolute value of the eigenvalues of the Hermitian matrix $G_{2}^{(2)}\left(t_{f} ; t_{i}, t_{1}, t_{1}^{\prime}\right)$ given by Eq. (78). This quantity which controls the error after one iteration is represented as a function of $t_{1}$ and $t_{1}^{\prime}$ for the KAM expansion of type B. By minimizing $g_{2}$, which is the norm of this operator, with respect to the free parameters $t_{1}$ and $t_{1}^{\prime}$, one reduces the error without having to determine the exact solution. Notice that $\left(t_{1}=0, t_{1}^{\prime}=0\right)$ is a local maximum of $g_{2}$ whereas $\left(t_{1}=0.5, t_{1}^{\prime}=0.5\right)$ is a saddle point. The point $\left(t_{1}=0.5, t_{1}^{\prime} \approx 0.22\right)$ corresponds to a minimum. The symmetry of Fig. 3 results from the pulse of Eq. (94) being symmetric.

Figure 4 displays the error $\Delta_{1}$ and the eigenvalue $g_{2}$ as a function of $t_{1}^{\prime}$ for the three types of one-iteration KAM expansions in the case $A=1, \epsilon=0.5$. The value of $t_{1}$ is chosen so that the optimum can be reached: $t_{1}=0.5$ for the type $\mathrm{B}$ and $t_{1}=0.7$ for the type $\mathrm{C}$ (recall that the type $\mathrm{A}$ features no $\left.t_{1}\right)$. One sees that the error can be reduced by more than one order of magnitude for the type B or C, and about half an order of magnitude for the type A after a single KAM iteration. It is also seen that the eigenvalue $g_{2}$ is a very accurate estimation of the error $\Delta_{1}$, which enables one to locate the optimal values of the free parameters. Note that the first order Magnus expansion corresponds to the particular value $t_{1}^{\prime}=0$ (i.e. the nonoptimized case) of the one-iteration type A KAM expansion.

We now turn to the next level of approximation for the Magnus expansion and the KAM expansion of type B. Recall that the first order and one-iteration expansions of these schemes yield comparable errors $\Delta_{1}$ for $A=$ 1. Figure 5 shows that the (nonoptimized) two-iteration KAM expansion performs better than the second order Magnus expansion by one to two orders of magnitude for $\Delta_{2}$. The error $\delta_{2}$ on the transition probability is also much smaller for the KAM expansion.

It is worth noting from the comparisons of Figs. 2 and 5 that the error $\Delta_{1}$ for the optimized one-iteration KAM expansion is comparable to the error $\Delta_{2}$ for the nonoptimized two-iteration KAM expansion of type B. This conclusion is not restricted to the type $\mathrm{B}$ and can be understood on the basis of the Campbell-Baker-Hausdorff formula as discussed in Sec. ID 4

If one optimizes the type B KAM expansion by choosing $t_{1}=0.5, t_{1}^{\prime}=0.22$ (as determined above from Fig. 3 ) and $t_{2}=0.66, t_{2}^{\prime}=0.8$, one gains another one to two orders of magnitude on $\Delta_{2}$.

From Fig. 5 one also deduces that the Dyson approach is not applicable in this context as the second order performs worse than the first order by both measures of the error. Notice that the transition probability predicted by the Dyson technique diverges, as is well-known, by lack of unitarity. In other words, the Dyson expansion does not allow one to refine the results of Fig. 2.

The two-iteration KAM expansion involves the operator $V_{2}(t)$ which is given by Eq. (34) or (36) as an infinite series of commutators. For two-level systems, this series can be computed explicitly and results in Eq. (90) with $n=1$. It is remarkable that the coefficient $a_{1}, b_{1}, c_{1}$ and $d_{1}$ are well-defined for all values of $\epsilon$, even larger than unity. In Fig. 5 we consider the cases where $V_{2}(t)$ is truncated to two commutators [i. e. the term $k=1$ of or$\operatorname{der} \epsilon^{0}$ and the term $k=2$ of order $\epsilon$ in Eq. (36)], and four commutators $(k=1, \cdots, 4)$. Note that this amounts to approximate the coefficients of Eq. (91) by polynomials (of order, respectively, 2 and 4 ) in $\epsilon \lambda_{1}(t)$. The case of two commutators performs better than the second-order Magnus expansion which is not surprising as it contains all the terms of order $\epsilon^{3}$. However, it performs worse than the KAM expansion with the infinite series by up to one order of magnitude. The case of four commutators is very close to the exact two-iteration KAM expansion for values of $\epsilon$ up to unity. The convergence with the number of commutators involved is indeed very fast. It has to be remarked that including commutators of higher orders 
in a well-defined manner as in the KAM algorithm is the main difference with the Magnus expansion (or any order by order expansion) and necessary to achieve superexponentiality. From a practical point of view the inclusion of more than one commutator is both convenient and highly profitable.

The fact that for the KAM expansion the order of the remainder be the exponential of an exponential $\left(\epsilon^{2^{n-1}}\right)$ implies that the accuracy is increased in a dramatic way with the number $n$ of iterations. In addition, the accuracy of a given $n$-iteration KAM expansion can still be enhanced, as illustrated here, and allows one to get closer to the next iteration of the algorithm by including through the simple variation of the parameters $t_{n}$ and $t_{n}^{\prime}$ more appropriate higher order terms.
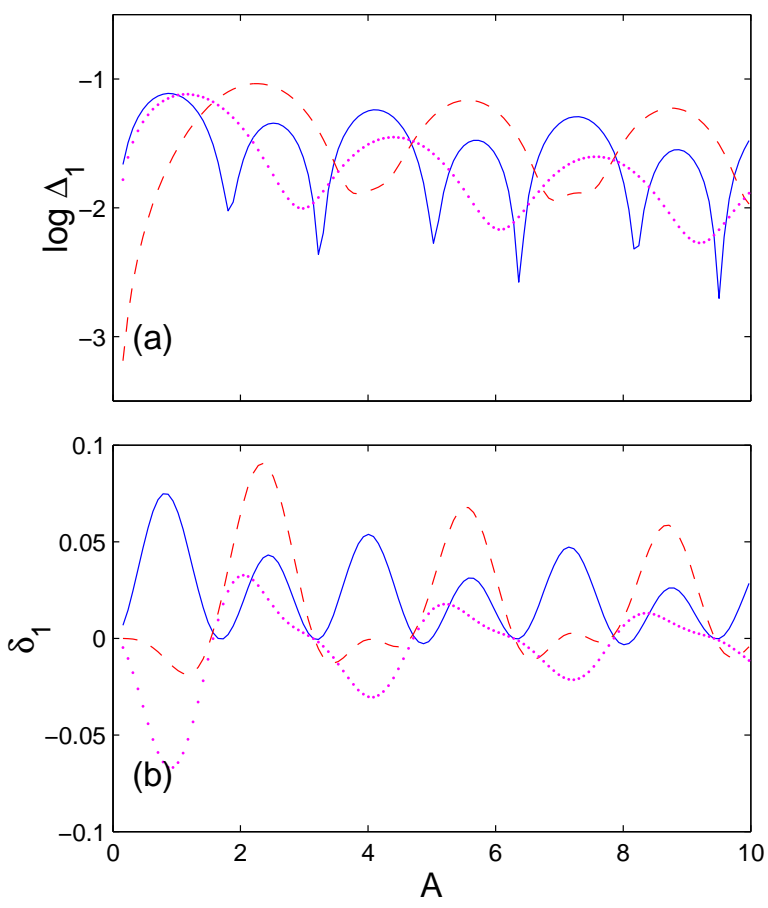

FIG. 1: Comparison of the first order Magnus expansion (dots), the one-iteration type B KAM expansion (solid line) and the one-iteration type C KAM expansion (dashed line) for $\epsilon=0.5$ and $t_{1}=t_{1}^{\prime}=0$ : (a) common logarithm of the error $\Delta_{1}$ and (b) error $\delta_{1}$ as a function of $A$. The one-iteration type A KAM expansion coincides in the case $t_{1}^{\prime}=0$ with the first order Magnus expansion. All quantities are dimensionless.

\section{CONCLUSIONS}

We have formulated perturbation theory in operator form 28 for time-dependent problems localized in time directly in the original Hilbert space by unitarily transforming the evolution operator.

We have compared formally and numerically various unitary perturbative schemes. The superiority of the KAM technique over the Magnus expansion, as well as
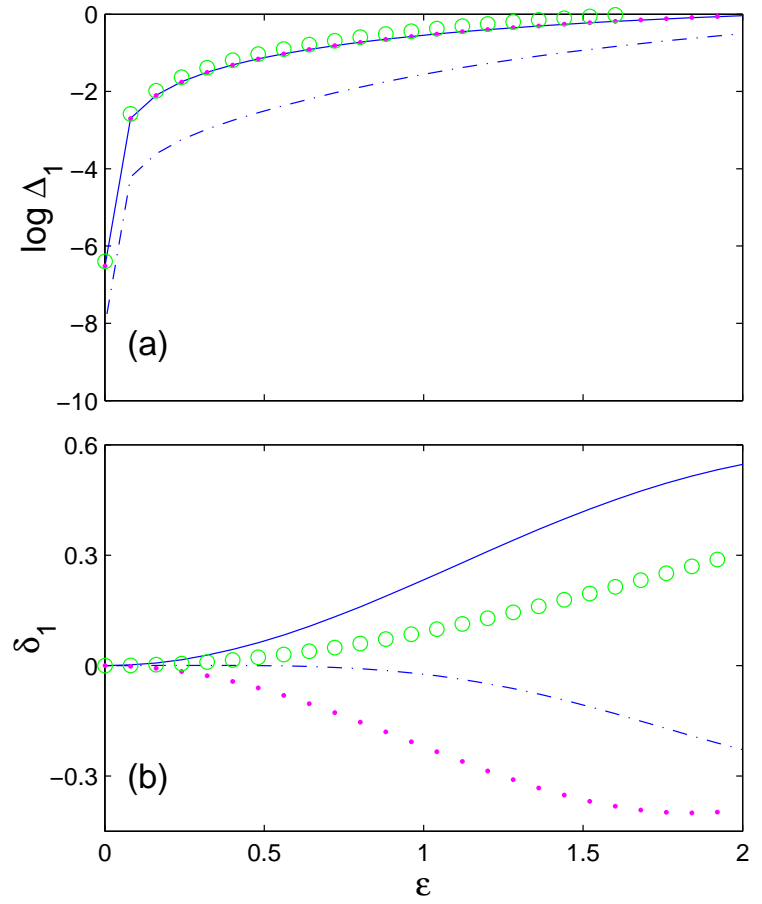

FIG. 2: Comparison of the first order Magnus expansion (dots), the first order Dyson expansion (circles), the oneiteration type B KAM expansion with $t_{1}=t_{1}^{\prime}=0$ ( solid line) and the optimized one-iteration type B KAM expansion with $t_{1}=0.5, t_{1}^{\prime}=0.22$ (dash-dot line) for $A=1$ : (a) common logarithm of the error $\Delta_{1}$ and (b) error $\delta_{1}$ as a function of $\epsilon$. All quantities are dimensionless.

the other methods, has been established owing to its superexponential character and the accuracy optimization. We have also shown that the Magnus expansion is recovered as a special case of the time-dependent Poincaré-Von Zeipel expansion (whose time-independent version coincides with the Rayleigh-Schrödinger expansion 25|).

The possibility to enhance the accuracy of a given level of approximation stems from the free parameters and the free operators that appear naturally in the formulation presented here. It allows one to significantly reduce the error with respect to the exact solution without its knowledge by the minimization of an eigenvalue.

The above considerations, illustrated here on a pulsedriven two-level system, are straightforwardly applied to more involved problems (see Ref. [30] for an application to the orientation and alignment of molecules).

\section{Acknowledgments}

The authors are grateful to G. Nicolis for helpful comments. This research was supported in part by the Belgian FNRS, the Action Concertée Incitative Photonique from the French Ministry of Research, the Conseil Régional de Bourgogne and a CGRI-FNRS-CNRS cooperation. 


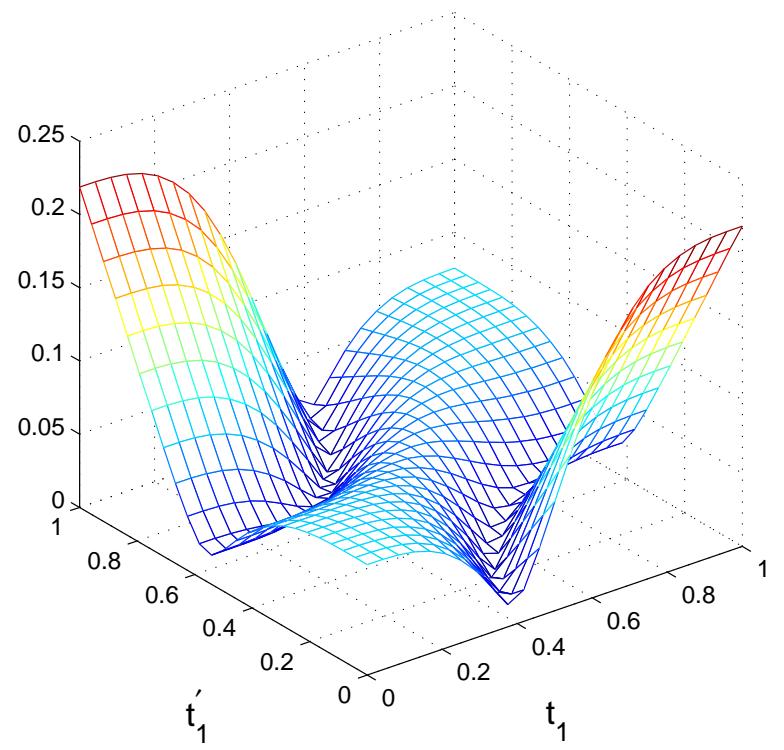

FIG. 3: Eigenvalue $g_{2}$ for the KAM expansion of type B as a function of $t_{1}$ and $t_{1}^{\prime}$, for $A=1$ and $\epsilon=0.5$. All quantities are dimensionless.

\section{APPENDIX A: TIME-DEPENDENT POINCARÉ-VON ZEIPEL EXPANSION}

The time-dependent Poincaré-Von Zeipel technique amounts to construct a unitary operator $T(t)$ which transforms the propagator $U_{H_{1}}\left(t, t_{0}\right)$ into a propagator $U_{H^{\mathrm{e}}}\left(t, t_{0}\right)$ according to

$$
T^{\dagger}(t) U_{H_{1}}\left(t, t_{0}\right) T\left(t_{0}\right)=U_{H^{\mathrm{e}}}\left(t, t_{0}\right),
$$

where $U_{H^{\mathrm{e}}}\left(t, t_{0}\right)$ is associated with an effective Hamiltonian $H^{\mathrm{e}}(t)$ containing contributions to every order in $\epsilon$

$$
H^{\mathrm{e}}(t) \equiv H_{0}(t)+\sum_{k=1}^{\infty} \epsilon^{k} D_{k}\left(t ; t_{k}\right)
$$

This propagator can be expressed in terms of $U_{H_{0}}\left(t, t_{0}\right)$ and unitary operators related to the partial Hamiltonians $\epsilon^{k} D_{k}\left(t ; t_{k}\right)$

$$
\begin{aligned}
U_{H^{\mathrm{e}}}\left(t, t_{0}\right) & =U_{H_{0}}\left(t, t_{0}\right) \exp \left[-i\left(t-t_{0}\right) \epsilon D_{1}\left(t_{0} ; t_{1}\right)\right] \\
& \times \cdots \exp \left[-i\left(t-t_{0}\right) \epsilon^{k} D_{k}\left(t_{0} ; t_{k}\right)\right] \cdots,
\end{aligned}
$$

provided the operator $D_{k}\left(t ; t_{k}\right)$ are defined as

$$
D_{k}\left(t ; t_{k}\right) \equiv U_{H_{k-1}^{\mathrm{e}}}\left(t, t_{k}\right) D_{k}\left(t_{k} ; t_{k}\right) U_{H_{k-1}^{\mathrm{e}}}\left(t_{k}, t\right) .
$$

Here $D_{k}\left(t_{k} ; t_{k}\right)$ is arbitrary but strictly of order $\epsilon^{0}$ (in contrast to the KAM case) and we set

$$
\begin{aligned}
U_{H_{n}^{\mathrm{e}}}\left(t, t_{0}\right) & \equiv U_{H_{0}}\left(t, t_{0}\right) \exp \left[-i\left(t-t_{0}\right) \epsilon D_{1}\left(t_{0} ; t_{1}\right)\right] \\
& \times \cdots \exp \left[-i\left(t-t_{0}\right) \epsilon^{n} D_{n}\left(t_{0} ; t_{n}\right)\right] .
\end{aligned}
$$
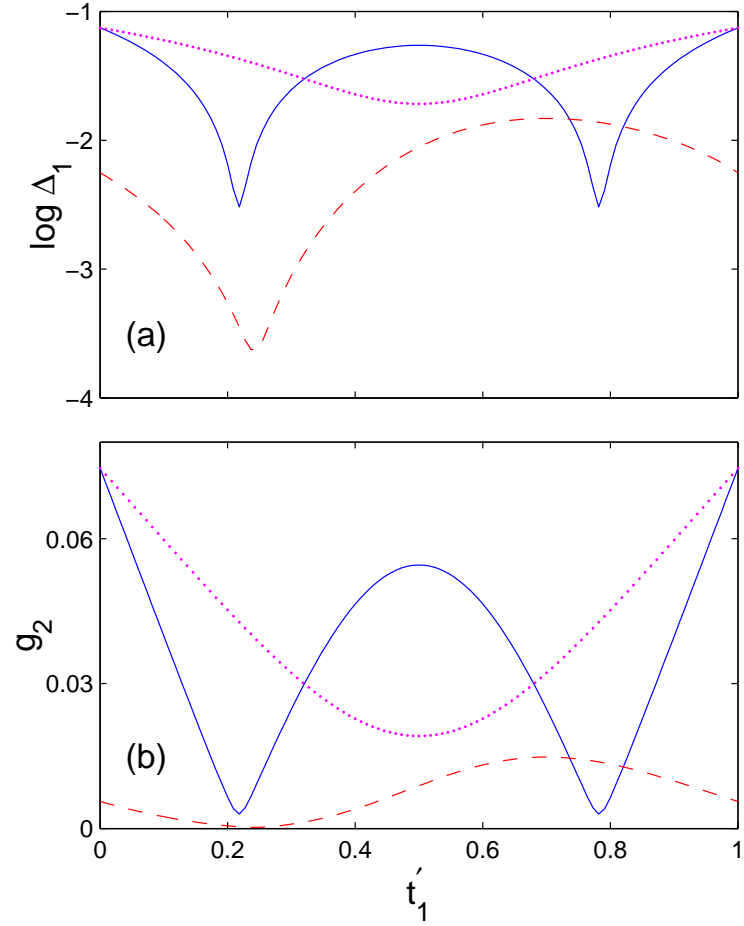

FIG. 4: Comparison of the one-iteration type A KAM expansion (dots), the one-iteration type B KAM expansion with $t_{1}=0.5$ (solid line) and the one-iteration type C KAM expansion with $t_{1}=0.7$ (dash-dot line) for $A=1$ and $\epsilon=0.5$ : (a) common logarithm of the error $\Delta_{1}$ and (b) eigenvalue $g_{2}$ as a function of $t_{1}^{\prime}$. All quantities are dimensionless.

The generator of the Poincaré-Von Zeipel transformation $T(t)$ is written as a power series of $\epsilon$-independent operators $W_{k}(t)$

$$
T(t) \equiv \exp \left(-i \sum_{k=1}^{\infty} \epsilon^{k} W_{k}(t)\right) .
$$

These operators satisfy the differential equations

$$
\frac{\partial}{\partial t} W_{k}(t)=V_{k}(t)-D_{k}\left(t ; t_{k}\right)+i\left[W_{k}(t), H_{0}(t)\right],
$$

where the expression for $V_{k}(t)$ cannot be given in a simple form for arbitrary $k$ and requires increasing algebra. The situation is analogous for the Magnus expansion or the Van Vleck expansion given below, and in contrast to the KAM expansion where the new effective perturbation has exactly the same form at each step [cf. Eq. [60)]. The general solution to Eq. (A7) reads (up to a term $U_{H_{0}}\left(t, t_{0}\right) B_{k} U_{H_{0}}\left(t_{0}, t\right)$ with $B_{k}$ any constant self-adjoint operator)

$$
W_{k}(t)=\int_{t_{k}^{\prime}}^{t} d u U_{H_{0}}(t, u)\left[V_{k}(u)-D_{k}(u)\right] U_{H_{0}}(u, t),
$$

where $t_{k}^{\prime}$ is arbitrary. Notice that this expression involves the unperturbed propagator unlike the KAM analogue which features an effective propagator [cf. Eq. (59)]. 

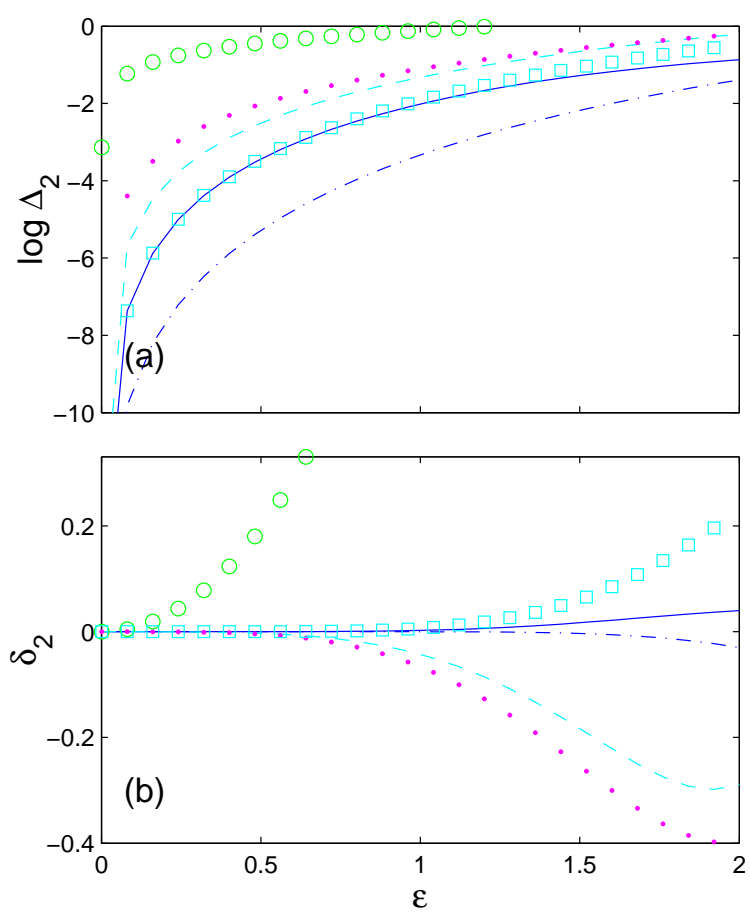

FIG. 5: Comparison of the second order Magnus expansion (dots), the second order Dyson expansion (circles), the twoiteration type B KAM expansion $\left(t_{1}=t_{1}^{\prime}=t_{2}=t_{2}^{\prime}=0\right.$ ) with the infinite series of commutators (solid line), two commutators (dashed line), four commutators (squares) and the optimized $\left(t_{1}=0.5, t_{1}^{\prime}=0.22, t_{2}=0.66, t_{2}^{\prime}=0.8\right)$ twoiteration type B KAM expansion (dash-dot line) for $A=1$ : (a) common logarithm of the error $\Delta_{2}$ and (b) error $\delta_{1}$ as a function of $\epsilon$. All quantities are dimensionless.

For the operator $D_{k}\left(t ; t_{k}\right)$ of Eq. (A4) to be strictly of order $\epsilon^{0}$, we are no longer entitled to choose $U_{H_{k-1}^{\mathrm{e}}}\left(t, t_{k}\right) V_{k}\left(t_{k}\right) U_{H_{k-1}^{\mathrm{e}}}\left(t_{k}, t\right)$ for any $k$ if that choice has already been made for a lower value of $k$. This choice can only be made once (for an arbitrary value of $k$ denoted $v$ ) and implies to take $D_{k}\left(t ; t_{k}\right) \equiv 0$ for the other values of $k$.

We then construct the $n$-th order unitary approximation to $T(t)$

$$
T_{n}(t) \equiv \exp \left(-i \sum_{k=1}^{n} \epsilon^{k} W_{k}(t)\right),
$$

and by Eqs. A1 and A3 obtain the $n$-th order Poincaré-Von Zeipel approximation to the propagator $U_{H_{1}}\left(t, t_{0}\right)$

$$
\begin{aligned}
U_{H_{1}}^{(n)}\left(t, t_{0}\right) & =T_{n}(t) U_{H_{0}}\left(t, t_{0}\right) \\
& \times \exp \left[-i\left(t-t_{0}\right) \epsilon^{v} D_{v}\left(t_{0} ; t_{v}\right)\right] T_{n}^{\dagger}\left(t_{0}\right),
\end{aligned}
$$

where $v$ is any integer between 1 and $n$ for which we have the possibility to choose either $D_{v}\left(t_{0} ; t_{v}\right) \equiv$ $U_{H_{0}}\left(t_{0}, t_{v}\right) V_{v}\left(t_{v}\right) U_{H_{0}}\left(t_{v}, t_{0}\right)$ or $D_{v}\left(t_{0} ; t_{v}\right) \equiv 0$.
It is interesting to note that Eq. A10 precisely reduces to the Magnus expansion if we take $D_{v}\left(t ; t_{v}\right) \equiv 0$ and $t_{k}^{\prime}=t_{0}$ for all $k$. In the general case there are up to $n+1$ free parameters

$$
\begin{aligned}
T_{n}(t) & \equiv T_{n}\left(t ; t_{v}, t_{1}^{\prime}, \cdots, t_{n}^{\prime}\right), \\
W_{n}(t) & \equiv W_{n}\left(t ; t_{v}, t_{1}^{\prime}, \cdots, t_{n}^{\prime}\right) .
\end{aligned}
$$

\section{APPENDIX B: TIME-DEPENDENT VAN VLECK METHOD}

The time-dependent Van Vleck technique is an order by order method where instead of transforming the original propagator into a final new propagator in a single step as in the Poincaré-Von Zeipel algorithm, one achieves this goal iteratively through a series of transformations $T_{k}(t)$ which reduce the size of correction terms from $\epsilon^{k}$ to $\epsilon^{k+1}$. This results in the $n$-th order Van Vleck expansion

$$
\begin{aligned}
& U_{H_{1}}^{(n)}\left(t, t_{0}\right)=T_{1}(t) \cdots T_{n}(t) U_{H_{0}}\left(t, t_{0}\right) \\
& \quad \times \exp \left[-i\left(t-t_{0}\right) \epsilon^{v} D_{v}\left(t_{0} ; t_{v}\right)\right] T_{n}^{\dagger}\left(t_{0}\right) \cdots T_{1}^{\dagger}\left(t_{0}\right),
\end{aligned}
$$

where $D_{v}\left(t_{0} ; t_{v}\right) \equiv U_{H_{0}}\left(t_{0}, t_{v}\right) V_{v}\left(t_{v}\right) U_{H_{0}}\left(t_{v}, t_{0}\right)$ or $D_{v}\left(t_{0} ; t_{v}\right) \equiv 0$, with $v$ an integer between 1 and $n$. Here we define

$$
T_{k}(t) \equiv e^{-i \epsilon^{k} W_{k}(t)},
$$

with $W_{k}(t)$ constructed as in Eq. (A8). There are up to $n+1$ free parameters entering the $n$-th order timedependent Van Vleck expansion.

The Van Vleck and Poincaré-Von Zeipel techniques differ because of the product of exponentials of $W_{k}(t)$ appearing in Eq. (BB1) instead of the single exponential of a sum of $W_{k}(t)$ in Eq. A10. As discussed above on the basis of the Campbell-Baker-Hausdorff formula this means that these algorithms differ at orders higher than the prescribed order.

\section{APPENDIX C: KAM EXPANSION IN THE INTERACTION REPRESENTATION}

In the framework of a perturbation theory, we stressed in Sec. 1 that the Magnus expansion had to be derived in the interaction representation. In the section above, the KAM algorithm was applied in the original representation. We show here that going to the interaction representation, applying the KAM technique and coming back to the original representation yields identically the same expansions as above despite the truncation at any finite order.

Given an Hamiltonian $H_{1}(t)=H_{0}(t)+\epsilon V_{1}(t)$ and the propagator $U_{H_{0}}\left(t, t_{0}\right)$ we consider the interaction representation with respect to $H_{0}(t)$. From Eq. (2) one deduces that $H_{1}^{\mathrm{i}}(t ; s)=H_{0}^{\mathrm{i}}(t ; s)+\epsilon V_{1}^{\mathrm{i}}(t ; s)$ with

$$
\begin{aligned}
H_{0}^{\mathrm{i}}(t ; s) & \equiv 0, \\
V_{1}^{\mathrm{i}}(t ; s) & \equiv U_{H_{0}}(s, t) V_{1}(t) U_{H_{0}}(t, s) .
\end{aligned}
$$


Furthermore, the unperturbed propagator is trivial

$$
U_{H_{0}^{\mathrm{i}}}\left(t, t_{0} ; s\right)=\mathbb{1} .
$$

It is worth pointing out that Eq. C1b suggests that $\epsilon V_{1}^{\mathrm{i}}(t ; s)$ is considered small with respect to $H_{0}^{\mathrm{i}}(t ; s)=0$. As a matter of fact, by virtue of Eq. (2b), it is with respect to $H_{0}^{\mathrm{i}}(t ; s)-i \frac{\partial}{\partial t}$ that $\epsilon V_{1}^{\mathrm{i}}(t ; s)$ is considered small (in a technical sense we need not specify here).

Applying the KAM algorithm with the identifications of Eqs. (C1b) and (C1c) leads to the expansion

$$
\begin{aligned}
U_{H_{1}^{\mathrm{i}}}^{(n)}\left(t, t_{0} ; s\right) & =T_{1}(t ; s) \cdots T_{n}(t ; s) U_{H_{n}^{\mathrm{ei}}}\left(t, t_{0} ; s\right) \\
& \times T_{n}^{\dagger}\left(t_{0} ; s\right) \ldots T_{1}^{\dagger}\left(t_{0} ; s\right) .
\end{aligned}
$$

Returning to the original representation with the help of Eq. (2a) one obtains a perturbative expansion for $U_{H_{1}}\left(t, t_{0}\right)$ that coincides exactly with the expansion $U_{H_{1}}^{(n)}\left(t, t_{0}\right)$ obtained directly in this representation

$$
U_{H_{0}}(t, s) U_{H_{1}^{\mathrm{i}}}^{(n)}\left(t, t_{0} ; s\right) U_{H_{0}}\left(s, t_{0}\right)=U_{H_{1}}^{(n)}\left(t, t_{0}\right) .
$$

This stems from the following identities readily derived on the basis of Eq. (10) and valid for any $k$

$$
\begin{aligned}
U_{H_{0}}(t, s) T_{k}(t ; s) U_{H_{0}}(s, t) & =T_{k}(t), \\
U_{H_{0}}(t, s) U_{H_{k}^{\mathrm{ei}}}\left(t, t_{0} ; s\right) U_{H_{0}}\left(s, t_{0}\right) & =U_{H_{k}^{\mathrm{e}}}\left(t, t_{0}\right) .
\end{aligned}
$$

[1] H. Stapelfeldt and T. Seideman, Rev. Mod. Phys. 75, 543 (2003).

[2] M. V. Berry, Proc. R. Soc. London A 429, 61 (1990).

[3] M. V. Berry and R. Lim, J. Phys. A 26, 4737 (1993).

[4] A. Joye, J. Phys. A 26,6517 (1993).

[5] K. Drese and M. Holthaus, Eur. Phys. J. D 3, 73 (1998).

[6] A. M. Dykhne, J. Exp. Theor. Phys. (U.S.S.R.) 41, 1324 (1961) [Sov. Phys. JETP 14, 941 (1962)].

[7] J. P. Davis and P. Pechukas, J. Chem. Phys. 64, 3129 (1976).

[8] A. Joye, H. Kuntz, and C.-Ed. Pfister, Ann. Phys. 208, 299 (1991); A. Joye, G. Mileti, and C.-Ed. Pfister, Phys. Rev. A 44, 4280 (1991).

[9] H. Sambe, Phys. Rev. A 7, 2203 (1973).

[10] J. S. Howland, Math. Ann. 207, 315 (1974).

[11] J. H. Shirley, Phys. Rev. 138, B979 (1965).

[12] J. C. A. Barata and W. F. Wreszinski, Phys. Rev. Lett. 84, 2112 (2000); J. C. A. Barata, Ann. I. H. Poincaré 2 2, 963 (2001).

[13] S. Guérin and H. R. Jauslin, Adv. Chem. Phys. 125, 147 (2003).

[14] D. Daems, A. Keller, S. Guérin, H. R. Jauslin and O. Atabek, Phys. Rev. A 67, 052505 (2003).

[15] D. Daems, S. Guérin, H. R. Jauslin, A. Keller and O. Atabek, Phys. Rev. A 68, 051402(R) (2003).

[16] J. Bellissard, Stability and Instability in Quantum Mechanics, in Trends and developments in the eighties, edited by S. Albeverio and Ph. Blanchard (World Scientific, Singapore 1985).

[17] M. Combescure, Ann. I. H. Poincaré 47, 63 (1987); M. Combescure, Ann. Phys. 185, 86 (1988).

[18] P. Blekher, H. R. Jauslin and J. L. Lebowitz, J. Stat. Phys. 68, 271 (1992).

[19] P. Duclos and P. Štovícek, Commun. Math. Phys. 177, 327 (1996); P. Duclos, P. Šťovíćek and M. Vittot, Ann. I. H. Poincaré 71, 241 (1999).
[20] W. Scherer, Phys. Lett. A 233, 1 (1997); W. Scherer, J. Math. Phys. 39, 2597 (1998).

[21] W. Magnus, Commun. Pur. Appl. Math. 7, 649 (1954).

[22] N. E. Henriksen, Chem. Phys. Lett., 312, 196 (1999).

[23] P. Pechukas and J. C. Light, J. Chem. Phys. 44, 3897 (1966).

[24] A. Iserles, Not. Am. Math. Soc. 49, 430 (2002).

[25] W. Scherer, J. Phys. A: Math. Gen. 27, 8231 (1994).

[26] W. Scherer, Phys. Rev. Lett. 74, 1495 (1995).

[27] F. Hausdorff, Ber. Säch. Akad. Wiss. Math. Phys. Kl. 58, 19 (1906).

[28] H. Primas, Rev. Mod. Phys. 35, 710 (1963).

[29] H. R. Jauslin, S. Guérin and S. Thomas, Physica A 279, 432 (2000).

[30] D. Sugny, A. Keller, O. Atabek, D. Daems, S. Guérin and H. R. Jauslin, submitted to Phys. Rev. A (2003).

[31] J. H. Van Vleck, Phys. Rev. 33, 467 (1929).

[32] W. H. Shaffer, H. H. Nielsen and L. H. Thomas, Phys. Rev. 56, 895 (1939).

[33] Notice that all the terms containing solely $H_{0}(t)$ are also given by a series as that of Eq. (6) where $H_{1}(t)$ is replaced by $H_{0}(t)$, and yield, as a consequence, the propagator $U_{H_{0}}\left(t, t_{0}\right)$. By going to the interaction representation we get rid of this particular series, and factor out this propagator right from the start.

[34] In Ref. 22] a time parameter is introduced through an interaction representation and shown to affect the final result. This is due to the fact that in Ref. [22] an additional approximation is made which assumes an impulsive character for the time-dependent perturbation.

[35] In a similar vein, on the basis of Eq. [66 one can define a small parameter $\epsilon m_{1}$ where $\epsilon$ is the perturbative (ordering) parameter [cf. Eq. [1b] and $m_{1}$ is the norm of $M_{1}\left(t, t_{0}\right)$. Notice from the definition given in Eq. (64) that this operator samples the perturbation $V_{1}(t)$ during its whole duration. 